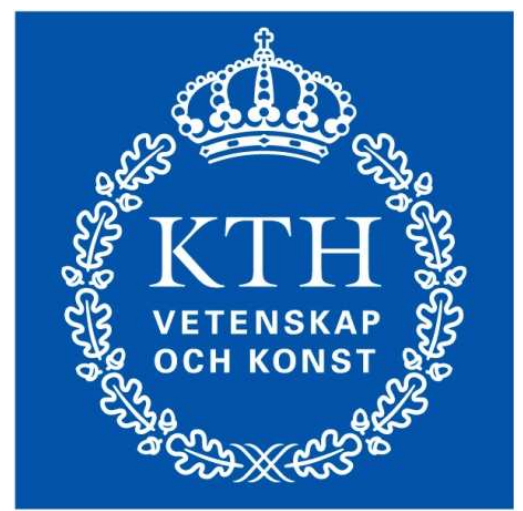

ROYAL INSTITUTE OF TECHNOLOGY

\title{
Optimal Design of Cost- and Energy-Efficient Scalable Passive Optical Backbone Networks
}

\author{
Farzad Abtahi
}

abtahi@kth.se 



\section{Abstract}

Advances in optical coherent transmissions and electrical compensation technologies have stimulated the exploration of novel optical network architectures. Filterless optical backbone networks (F-OBNs) eliminate or minimize the usage of active photonic reconfigurable components, which is also referred to as passive OBN. By introducing passive splitters and combiners to interconnect the fiber links, this type of networks have been proposed as a cost- and energy-efficient alternative to active optical switching networks.

However, F-OBN suffers from a constraint on wavelength reuse due to its broadcast nature. Consequently, this architecture always requires more resources, i.e. higher number of wavelengths, than the active optical switching networks. To address this issue, another passive approach for optical core network, i.e., semi-filterless OBN (SF-OBN) has been introduced. By utilizing passive colored components, e.g., Fiber Bragg Grating (FBG), $\mathrm{red} / \mathrm{blue}$ filters, etc., at some selected nodes, the SF-OBN is able to improve the wavelength usage while keeping the similar level on cost and energy consumption as FOBN.

In this thesis project, an optimization model for wavelength assignment and filter placement in SF-OBN has been developed. F-OBN can be considered as a special case without any filter in SF-OBN. Using integer linear programming (ILP) formulation, the model aims to minimize the total number of wavelengths required in the network given the number of filters.

Furthermore, wavelength usage, cost and energy consumption in active optical switching, F-OBN and SF-OBN have been compared in order to evaluate the performance of the each network architecture. 


\section{Acknowledgment}

First of all, I would like to show my deep gratitude to Prof. Lena Wosinska, head of Optical Networks Laboratory for providing me the opportunity to work on this project and encouraging me during my thesis.

I would like to express my grateful thanks to my supervisor Dr. Jiajia Chen. This thesis would not have been possible without her help, and guidance. Working with them was a pleasure and great experience in my future career and I wish to extend my warmest thanks to all those who helped me with my work.

Thanks to my lovely wife Sahar who has been a true support whenever I needed and motivated me with her sweet words during my research work.

Finally, I would like to thank my family whose lovely and unconditional support has always helped me finding my path. 


\section{Contents}

Abstract...

Acknowledgment ......................................................................................................................ii

List of Figures …................................................................................................................... vii

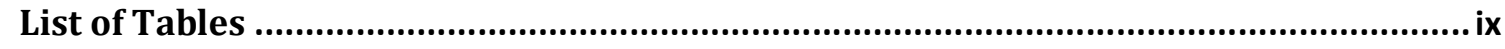

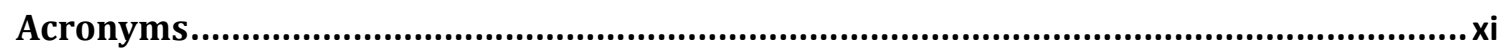

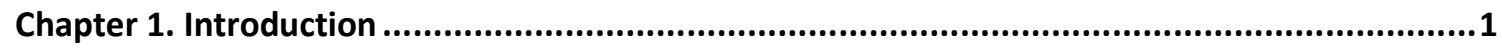

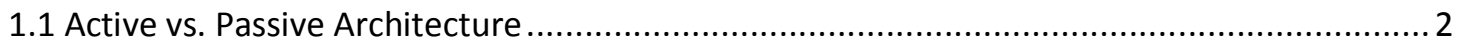

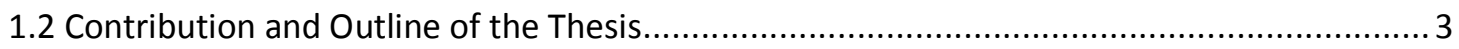

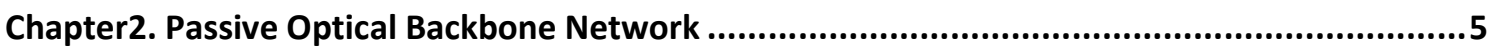

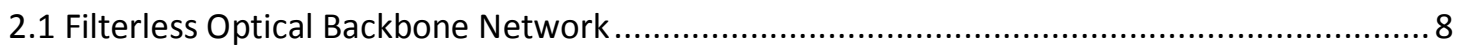

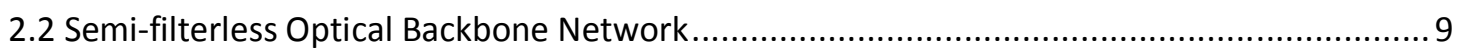

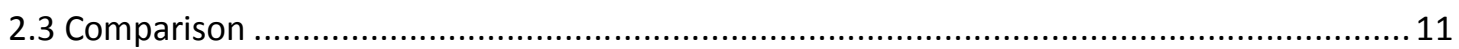

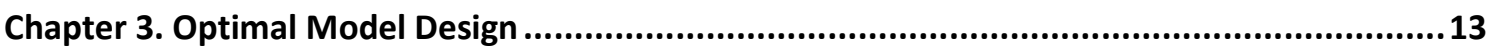

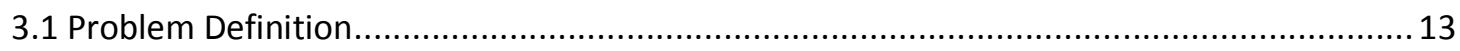

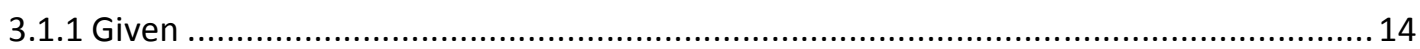

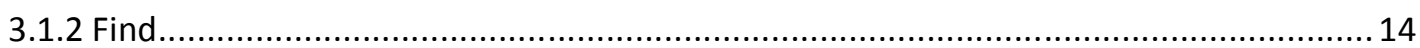

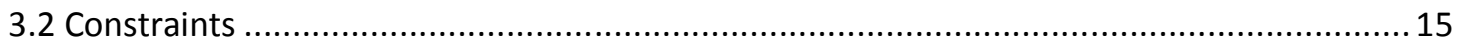

Chapter4. Performance Evaluation ........................................................................................

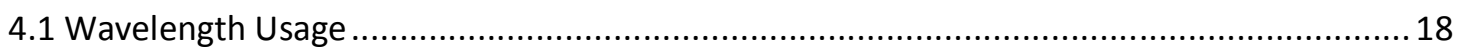

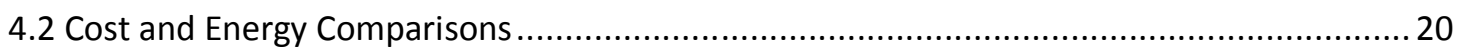

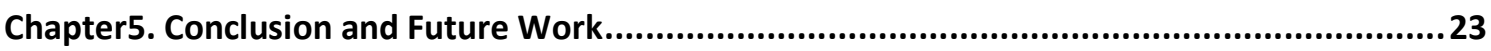

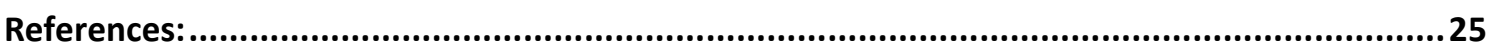

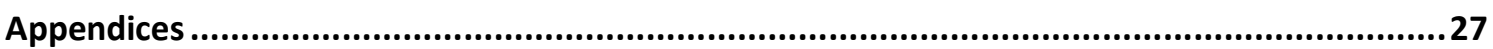




\section{List of Figures}

Figure 1. Photonic switched optical network using WSS-ROADM [7] ................................. 2

Figure 2. Flow of passive optical backbone network design problem..................................... 5

Figure 3. Laser effect in passive optical network................................................... 6

Figure 4. Physical topology (a) and fiber link interconnection design (b) for 7-node German

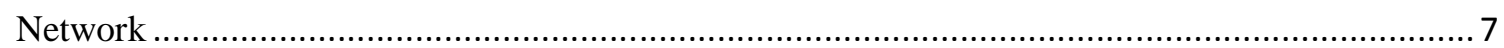

Figure 5. Example of routing step in a single fiber tree of 7-node German network ....................8

Figure 6. A sample filterless network with 6 nodes ................................................ 9

Figure 7. Semi-filterless architecture ....................................................................... 10

Figure 8. An illustrative example of the defined parameters ............................................... 14

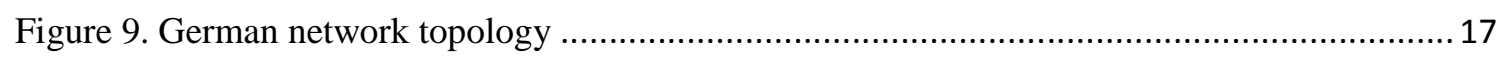

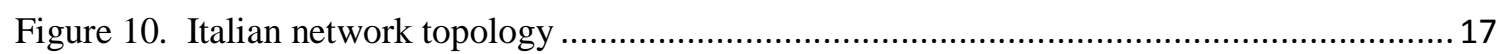

Figure 11. Wavelength usage vs. number of filters in 10-node Italian network (a) and 7-node German network (b) ...................................................................................... 18

Figure 12. Wavelength utilization comparisons for active and passive architectures ...................19

Figure 13 Cost (a) and energy consumption (b) comparisons for active and passive architectures. 22 


\section{List of Tables}

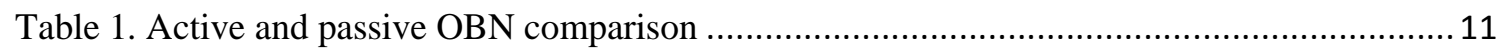

Table 2. Normalized values for cost and energy consumption in arbitrary unit (a.u.)[11] ............. 20

Table 3. The number of components in the German and Italy networks.....................................2 21 


\section{Acronyms}

DCF Dispersion Compensating Fiber

DCM Dispersion Compensating Module

EDFA Erbium Doped Fiber Amplifier

F-OBN Filterless Optical Backbone Network

HDTV High-definition television

IPTV Internet Protocol television

OBN Optical Backbone Network

OLA Optical Line Amplifier

OXC Optical Cross Connect

ROADM Reconfigurable Optical Add/Drop Multiplexers

SF-OBN Semi-filterless Optical Backbone Network

WSS Wavelength Selective Switch 


\section{Chapter 1. Introduction}

The telecom industry has been facing challenges in coping up with increasing amount of traffic generated by users requesting access to bandwidth-heavy services e.g. Internet Protocol television (IPTV), multicast applications such as High-definition television (HDTV), online gaming, etc. In order to satisfy this growing demand of internet users, new transmission technologies have been introduced in the optical networking area $[1,2]$.

Optical backbone network (OBN) architectures can be categorized into two main groups, according to the usage of active or passive network elements described as follows:

(1) Active optical backbone network

An active OBN architecture utilizes active components to switch individual wavelengths and spectrums into separate paths for specific routing of information. Hence, the active optical network has high wavelength utilization, with a relatively higher cost of implementation. In this type of network the content which is destined to a particular device is only delivered to that specific node.

(2) Passive optical backbone network.

Passive OBN architecture does not contain any active switching components for fiber link interconnection. Instead, it utilizes optical splitters/combiners and colored filters.

In a passive network, the traffic is sent from splitters/combiners of the source node to all directly connected nodes. The transmission continues along all available paths till to a node where no splitter or combiner exists. Therefore, all the nodes along the path of broadcasted signal can receive the data which might not be destined to them.

This study is focused on two newly proposed architectures of passive OBN: filterless OBN (F-OBN) and semi-filterless OBN (SF-OBN) architectures [3-5].

Active and passive OBN architecture have their advantages and drawbacks. While active networks are expensive to deploy, they offer networks with higher wavelength utilization and flexibility.

On the other hand, passive OBN does not need active reconfigurable components for switching function; hence, they are more cost and energy efficient. Moreover, passive architectures have lower failure rate since passive components have fewer failure modes 
than the active devices. As a result, they are considered more reliable than the active networks and can be seen as an attractive proposition for the market to support tremendous traffic demands. Beside the advantages, passive architectures reduce the investment cost of the network at the expense of greater wavelength usage.

Recent progresses in optical transmission technologies, like advanced modulation formats, electronic dispersion compensation and tunable transceivers, have motivated the exploration of novel optical network architectures [6]. The two recently proposed passive OBN architectures, filterless and semi-filterless are evaluated in this thesis study. These networks offer reliable, cost-effective and energy-efficient architectures compared with networks based on active photonic reconfigurable components. As the main contribution in this thesis, a novel optimal model is proposed to minimize the wavelength usage in the SFOBN architecture by optimizing the placement of given number of filters. A novel model based on integer linear programming (ILP) is proposed for medium size network topologies.

\subsection{Active vs. Passive Architecture}

Photonic switched optical network as an active optical backbone network architecture, utilizes optical cross connects (OXCs) or reconfigurable optical add/drop multiplexers (ROADMs) to interconnect links in intermediate nodes. Figure 1 demonstrates the photonic switched network using ROADMs with wavelength selective switch (WSS) $[1,7]$. Fiber links in the following figure are assumed to be bi-directional.

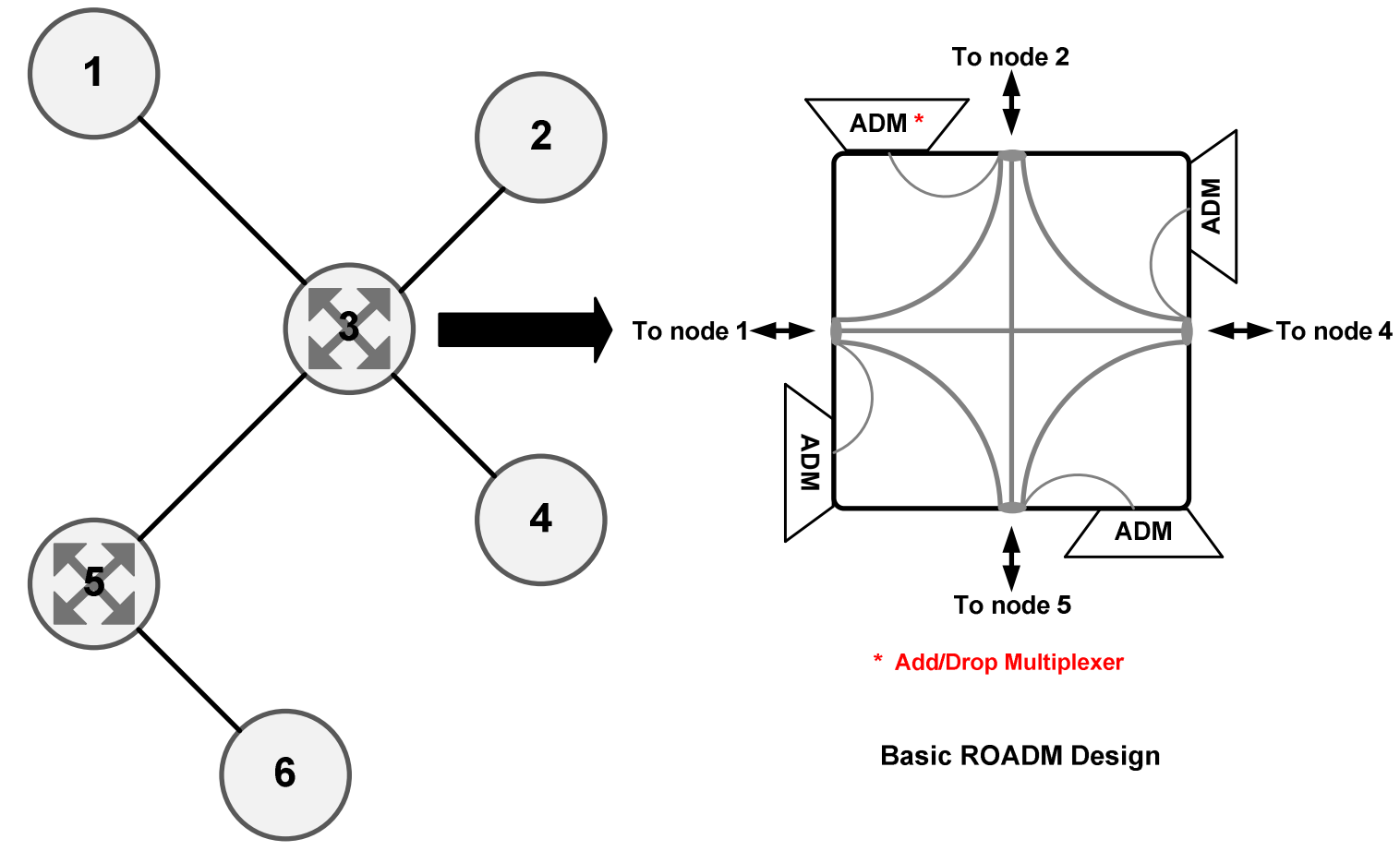

Figure 1. Photonic switched optical network using WSS-ROADM [7] 
On the other hand, the passive architecture utilizes passive splitters and combiners along with other passive elements in order to interconnect the fiber links in intermediate nodes in core networks. The passive devices are very simple and their costs are significantly lower than the active components. Due to passive elements usage in the passive networks, the signal of the lightpaths cannot be terminated in their intended destination and they are broadcasted through the other fiber links in the networks. The broadcast nature implies some constraints on fiber interconnection design in passive OBN. These constraints are laser loop, system reach and wavelength reuse. The following chapter describes the passive optical network architecture in details. In comparison with the active OBNs, the passive OBNs are simple and cost efficient if carefully designed.

\subsection{Contribution and Outline of the Thesis}

This thesis project proposes an optimal design of passive optical backbone network (OBN) by using integer linear programming (ILP). ILP is used to formulate wavelength assignment for lightpath demands and filter placement in order to increase wavelength utilization in passive OBNs. The main objective of the model is to minimize the wavelength usage. The model is solved for a number of network topologies. The obtained results confirm that a proper design of SF-OBN can offer significant reduction on the number of wavelengths, in comparison with F-OBN.

This thesis work was done as a part of a joint project between Royal Institute of Technology (KTH), École de technologie supérieure (ÉTS) and Ciena Corporation. Utilizing the results for active and filterless networks which are achieved from the project contributors, a comparison of cost and wavelength utilization in optical networks based on active switching, filterless and semi-filterless approach is provided. The results can be useful on both metro and backbone networks.

Background of this subject has already been provided and the remainder is organized as follows:

Chapter 2 gives an explanation about passive OBNs, including both filterless and semifilterless approaches. Furthermore, in this chapter, a comparison between F-OBN and SFOBN is carried out.

Chapter 3 presents the optimal network design for wavelength assignment and filter placement in passive optical backbone networks.

Chapter 4 shows the performance evaluation and comparison results of passive and active optical network architecture.

Chapter 5 presents the conclusion and future work of our study. 


\section{Chapter2. Passive Optical Backbone Network}

Passive optical backbone network (OBN) architectures have been proposed [3] as a costeffective, energy-efficient and reliable alternative to active optical switching network. They eliminate or minimize the usage of active photonic reconfigurable components and introduce passive splitters and combiners for fiber links interconnection. This chapter describes the two novel passive OBN architectures namely, filterless and semi-filterless networks [3, 4].

The network design problem of passive OBN takes some input parameters and constraints into account during each step in the design process. The solution is divided into three steps. The output of each step is used by the next steps as an input. Figure 2 illustrates a passive OBN design framework which consists of the following steps [5]:

1- Fiber interconnection

2- Routing

3- Wavelength assignment and filter placement (only for semi-filterless network)

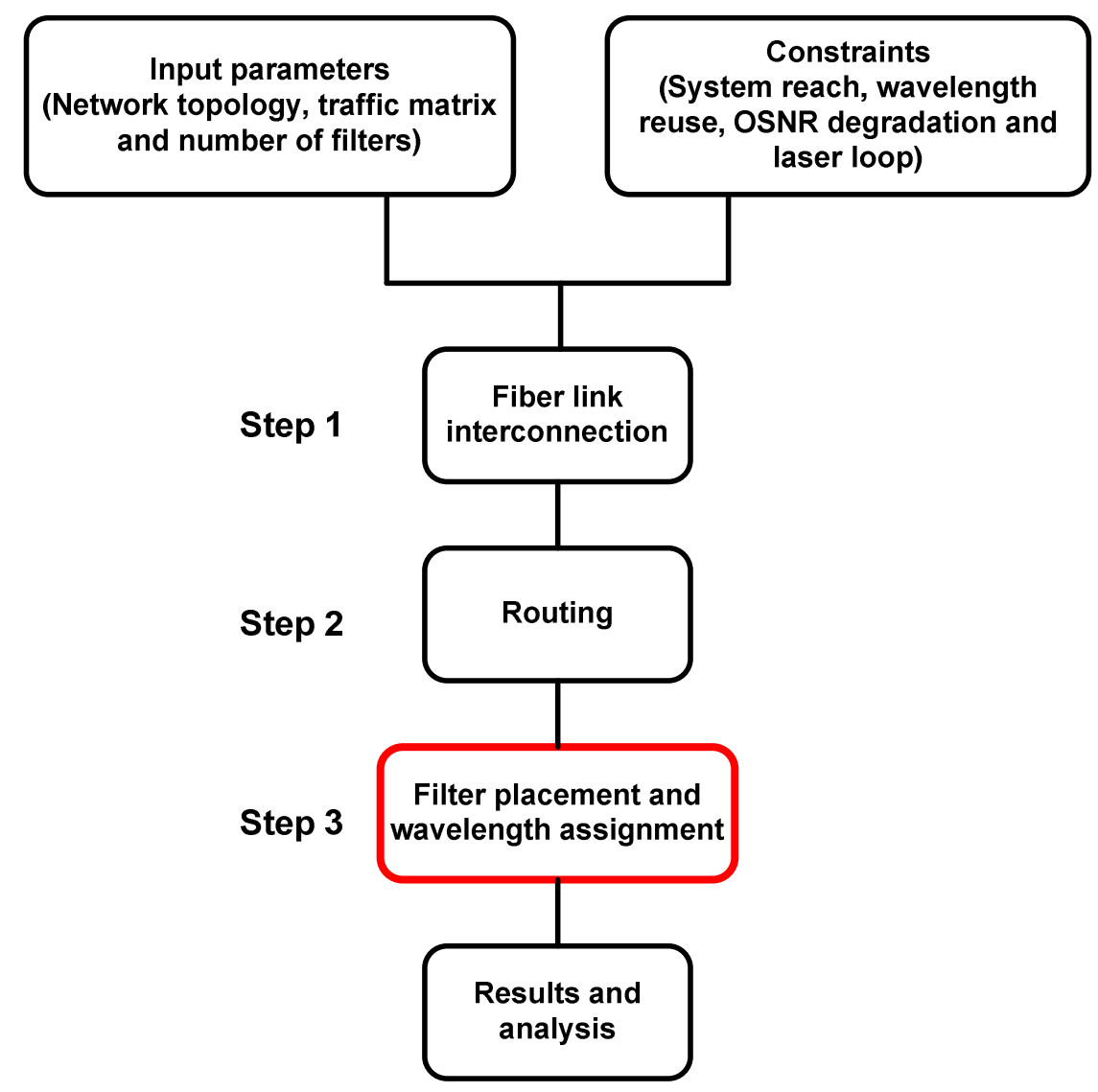

Figure 2. Flow of passive optical backbone network design problem 
In a passive optical network design, the following main constraints should be taken into consideration $[1,6]$ :

1. Wavelength-continuity constraint:

In the absence of wavelength conversion, a lightpath should operate on the same wavelength across all fiber links throughout its path.

2. Wavelength reuse constraint:

Two different cases should be taken into consideration:

- When two lightpaths share a common link, a conflict occurs between related wavelengths. Therefore different wavelengths should be assigned to each lightpath.

- Another scenario is when two lightpaths do not share a common link, but the signal continuation of a lightpath can affect the other one.

3. Laser loop constraint:

As mentioned earlier, fiber loops should be avoided because amplifier gain through the fiber links produces a laser effect. Figure 3 illustrates the laser effect which is created in closed loop.

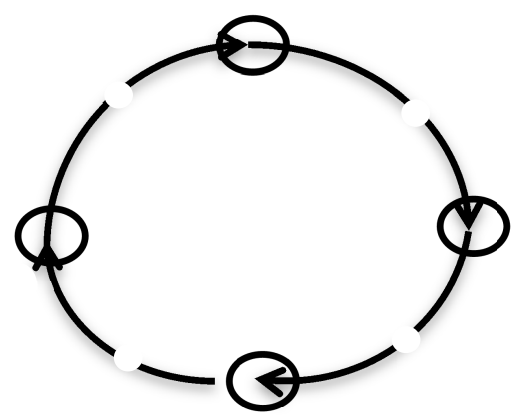

Figure 3. Laser effect in passive optical network

4. OSNR degradation constraint:

Optical signal to noise ratio (OSNR) has direct relation with bit error rate (BER), where BER is the ultimate value to measure the quality of transmission. Higher OSNR means lower BER which equals to fewer errors in transmission.

5. System reach constraint:

This is a physical layer constraint which defines the maximum distance for any root-leaf combination in a fiber tree. Longer system reach leads to OSNR degradation and higher BER. 
Considering these constraints, design of a passive OBN is divided into the following steps:

The first step is fiber link interconnection. The objective of this step is to provide connectivity for all nodes in a way that all of them can be physically accessible by any other nodes in the network. Network topology, traffic matrix and the number of filters are provided as input parameters for the design problem.

The output of this step gives information about number of fiber trees, splitters or combiners and their places in the network. Figure 4 shows the physical topology for a 7 node German network and one example of its fiber link interconnection design. In Figure 4.b fiber trees are represented by different colors.

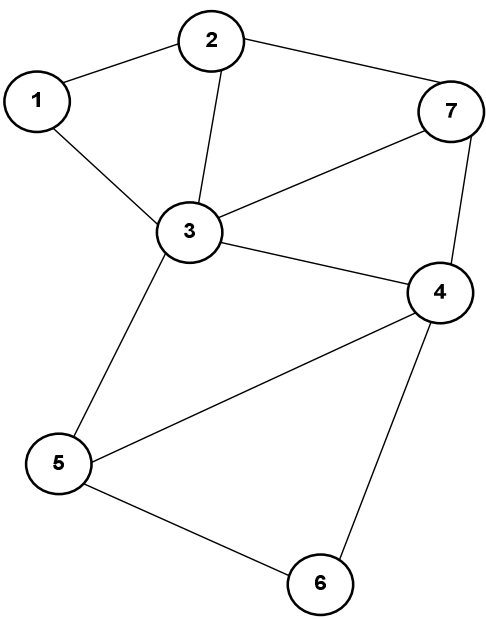

a

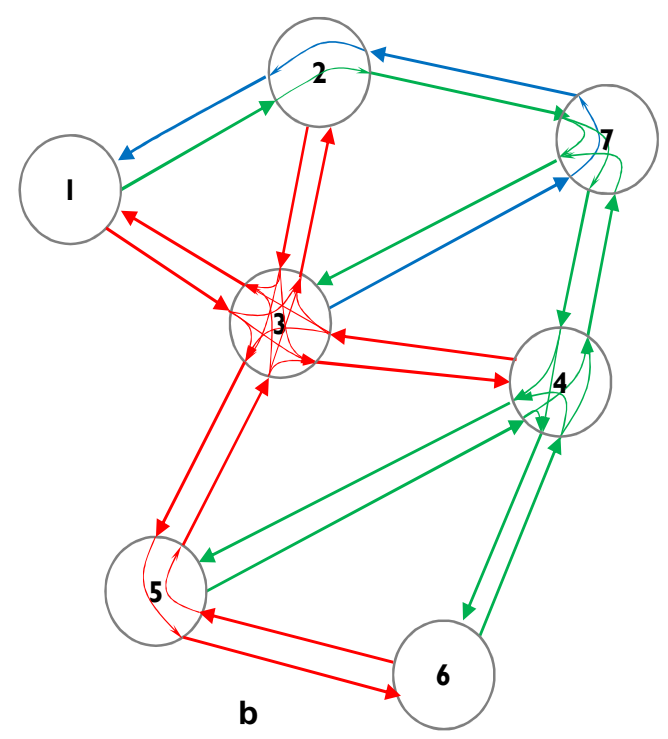

b

Figure 4. Physical topology (a) and fiber link interconnection design (b) for 7-node German Network

Fiber interconnection design (Figure 4.b) is used as an input for the routing step in addition to the other mentioned input parameters, e.g. traffic matrix. The objective of this step is to find a proper route for each request in the optical network. In this step the shortest path routing algorithm is used to find the best path for each demand. Each fiber tree can be treated separately since they are independent from each other. As an example, one of the fiber trees of the 7-node German network has been selected to show the routing step. Figure 5 shows the routing of the lightpaths (LPs) in the selected fiber tree. In this figure the splitters and combiners can be seen clearly in intermediate nodes. The white circles represent the combiners and the black ones are the splitters. 


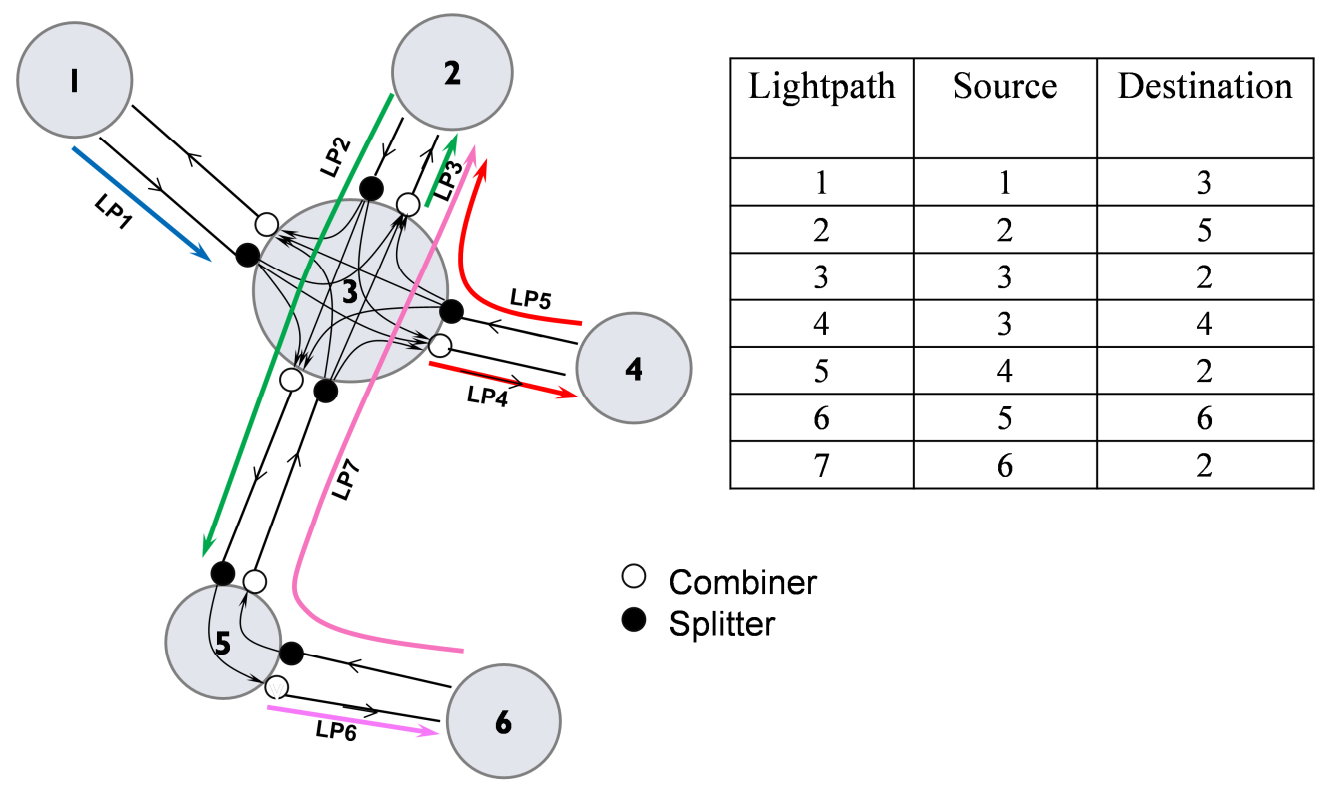

Figure 5. Example of routing step in a single fiber tree of 7-node German network

The last step, wavelength assignment and filter placement, is the main focus of this study the details of which is described in the following subsection. After finding the routes for the demands in the previous step, the wavelengths should be assigned. Furthermore, filter placement, if applicable, needs to be determined, where the purpose of each filter is to terminate specific wavelength signal at its destination. An optimal model for this step is proposed in this thesis in order to increase the resource utilization and decrease the cost of passive OBN by using integer linear programming (ILP). In the following of this chapter, two novel passive OBN, filterless and semi-filterless approach will be described.

\subsection{Filterless Optical Backbone Network}

Filterless optical backbone networks (F-OBNs) have been introduced in [3, 6].

The F-OBN aims to eliminate or minimize the usage of active photonic switching components. Instead of using active components, it interconnects the fiber links in intermediate nodes by splitters and combiners. Hence, F-OBN can be considered more cost-effective and energy-efficient compared to active architecture.

The broadcast nature in F-OBN which is inherited from passive OBN, applies some constraints on wavelength reuse in the network. It means that, in this passive architecture the assigned wavelength to a lightpath goes further than the intended destination node. Therefore the wavelength cannot be reused for other demands in a given fiber tree (see Figure 6). As a result an F-OBN solution may require considerably higher number of wavelengths as compared to the active networks to support a certain traffic demand. 


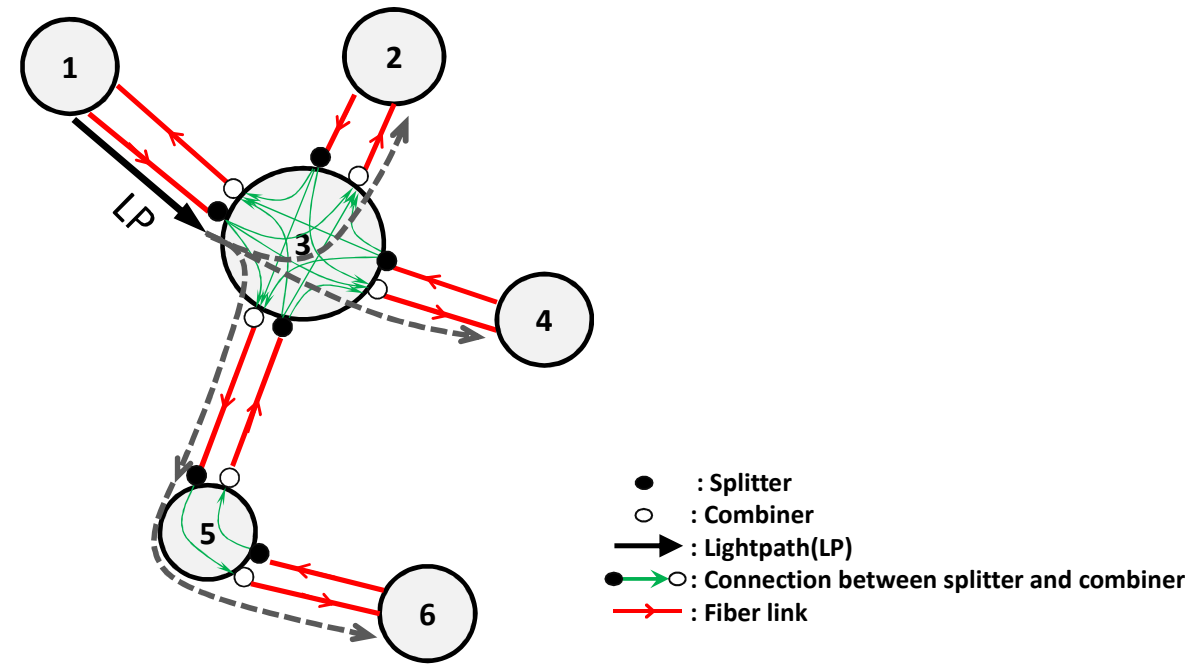

Figure 6. A sample filterless network with 6 nodes

As it is depicted in Figure 6, the destination of lightpath LP is node 3, but the signal of LP is not terminated at the destination node and it is broadcasted through other links in the network (the dashed line). Therefore the assigned signal for lightpath LP cannot be used for other demands on those links, as it may result in collision.

The semi-filterless network has been introduced as an extension of filterless architecture which could improve wavelength utilization. In the next sub-chapter, the semi-filterless optical backbone network architecture and its characteristics are illustrated.

\subsection{Semi-filterless Optical Backbone Network}

The semi-filterless optical backbone network (SF-OBN) has been proposed in $[4,5]$ as an extension of F-OBN which improves resource utilization, e.g., reduces the number of wavelengths required. The semi-filterless network takes benefit of passive colored components e.g., Fiber Bragg Gratings (FBG), red/blue filters, etc. SF-OBN utilizes nonbroadcast property of the colored components in order to solve wavelength reuse constraint inherited from the filterless architecture (see Figure 7). Therefore the SF-OBN can increase wavelength utilization at a relatively lower deployment cost compared to the filterless network.

Figure 7 demonstrates how the broadcast characteristics of F-OBN have been diminished via filter placement in some selected nodes in the SF-OBN architecture. The splitters and combiners were defined as outgoing and incoming contacts for the nodes. In Figure 7, Node 5 has two incoming contacts from Node 3 and 6, as represented with black circles, and two outgoing contacts to Node 3 and 6 , as depicted with white circles. The directed fiber link from node 1 to node 3 is referred to as a parent of links between node 3-2, 3-4, 3- 
5 and 5-6. The green lines inside node 3 represent the connections between splitters and combiners. Obviously, it is more efficient to place the filters to drop the wavelength at the incoming contacts than at the outgoing contacts. As it is shown in Figure 6, without any filters all the lightpaths starting from node 1 and passing the 1-3 link continue through all the child links, even though some of the lightpaths being addressed to node 3 could be dropped there. The signal of LP goes from 1 to 3, however after reaching its destination the signal is not terminated and it is broadcasted through child links in the fiber tree. On the other hand, in the semi-filterless network, the signal of lightpath LP is terminated by placing a colored passive filter at the incoming contact of Node 3 connecting to Node 1, and the wavelength of LP can be reused for other demands in child links (see Figure 7).

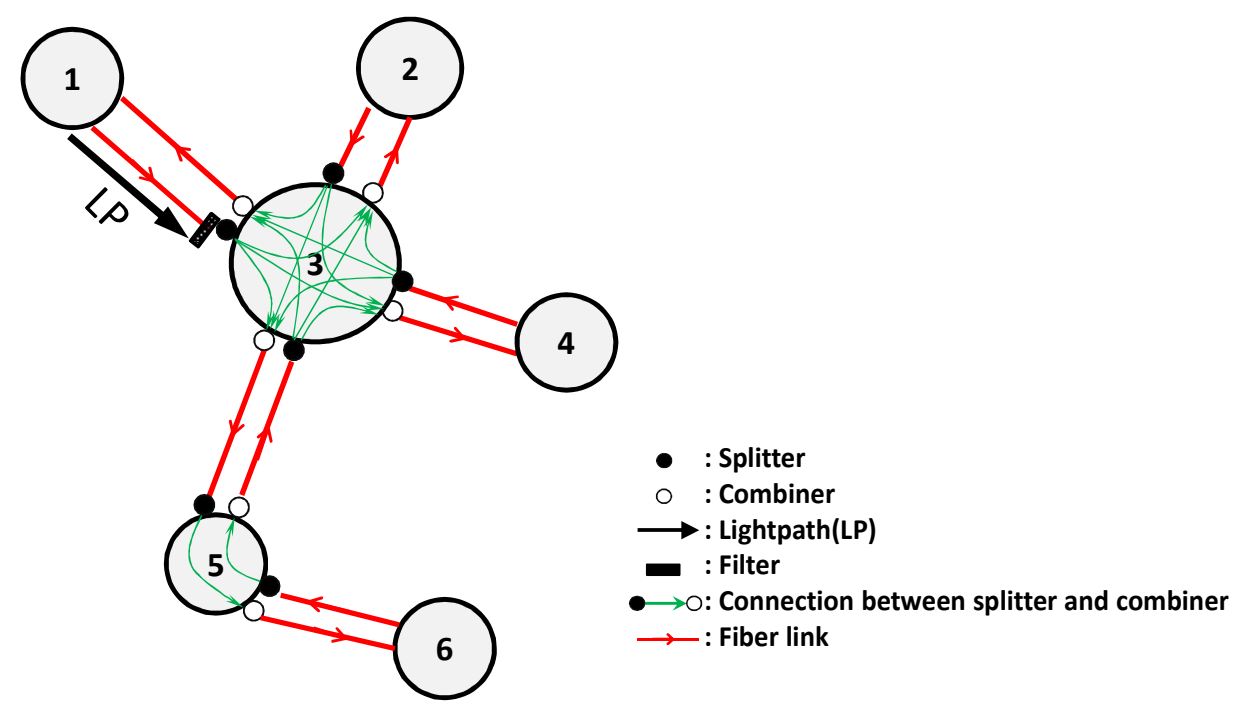

Figure 7. Semi-filterless architecture

As it is illustrated in the Figure 7, utilizing passive filters in semi-filterless approach can easily solve the wavelength reuse problem in filterless architecture and increase resource utilization in the network. By a relatively low increase in the deployment costs, the number of used wavelengths in the network can be decreased and consequently the capacity of passive networks increases.

The following section gives brief comparison between filterless and semi-filterless architecture. 


\subsection{Comparison}

Table 1 depicts that passive architectures are more desirable than the active networks in terms of capital investment cost and energy consumption, due to the usage of passive components in the network. Furthermore, in terms of reliability passive OBN architectures have better results than active photonic switch, since passive components have fewer failure modes.

On the other hand, when it comes to wavelength utilization and flexibility active networks stand on top. Since the SF-OBN utilizes colored passive components, in terms of flexibility it is placed after active photonic and F-OBN. The comparison between these architectures will be shown in detail in chapter 4 .

Table 1. Active and passive $\mathrm{OBN}$ comparison

\begin{tabular}{l|c|c|c} 
& Filterless solution & $\begin{array}{c}\text { Semi-filterless } \\
\text { solution }\end{array}$ & $\begin{array}{c}\text { Active photonic } \\
\text { switch }\end{array}$ \\
\hline Wavelength utilization & - & + & ++ \\
\hline Cost & ++ & ++ & -- \\
\hline Energy consumption & ++ & ++ & ++ \\
\hline Flexibility & + & - & + \\
\hline Reliability & ++ & ++ & + \\
\hline
\end{tabular}




\section{Chapter 3. Optimal Model Design}

In this chapter the Integer linear programming (ILP) model is presented for the wavelength assignment and filter placement problem in the design of passive optical backbone networks (OBN).

ILP is a mathematical optimization or feasibility program for determining a way to achieve the best outcome in a given optimization model. In an ILP model, some or all of the variables are restricted to be integers and represented as linear relationships. In the optimal model designed as a part of this thesis, a special case of linear programming was considered, in which unknown variables are binary [8]. SF-OBN architecture design consists of three steps [5]:

(1) Fiber interconnection

(2) Routing

(3) Wavelength assignment and filter placement

Heuristic solutions for wavelength assignment and filter placement are provided in [7] for filterless and in [5] for semi-filterless optical backbone networks. This thesis study proposes an ILP model for step 3 to minimize number of wavelengths by solving the placement problem of a set of filters in the network. The preliminary results are presented in [9]. The proposed optimal model can be taken as a point of reference for other models and algorithms in order to assess their efficiency of wavelength utilization.

In the following chapter the ILP formulation for SF-OBN is presented. The objective of the ILP model is to minimize the number of wavelengths by placing the given number of filters on some selected nodes in the network. Consequently, filter placement increases the wavelength utilization in semi-filterless network compared with the filterless solution.

\subsection{Problem Definition}

The objective of the proposed ILP model is to minimize the total number of wavelength usage in the network by placing a given amount of passive filters in some selected nodes. This model can also be used to optimize the number of wavelengths in F-OBNs by setting the number of filters in the model equal to 0 . The problem formulation and ILP model is shown below.

Problem formulation in ILP is expressed by the following sets of parameters. The first set is given, which contains all the problem input parameters. The find set includes the problem variables, values of which should be determined. By applying the constraints to the given set of parameters, solution of the variables can be obtained. 


\subsubsection{Given}

$D$ : the set of demands along with their routing information.

$w$ : The set of available wavelength for each fiber link.

$\boldsymbol{G}(\boldsymbol{N}, \boldsymbol{E})$ : the physical topology consists of the set of nodes $N$ and the set of fiber links $E$ where $l \in E$ denotes one fiber link.

$N F$ : the number of filters that need to be placed.

$\boldsymbol{P}_{l}$ : the set of parent links of $l$ where $P_{l} \subset E$ and $\mathrm{p}$ is used to index the parent links i.e., $p \in P_{l}$.

$\boldsymbol{K}_{p, l}$ : the set of demands which is routed through p but not $l$ where $K_{p, l} \subset D$.

$\boldsymbol{T}_{p, l}$ : the set of demands which is routed through $p$ and ended before $l$ where $T_{p, l} \subset D$.

Figure 8 illustrates the given input parameters in a simple fiber tree:

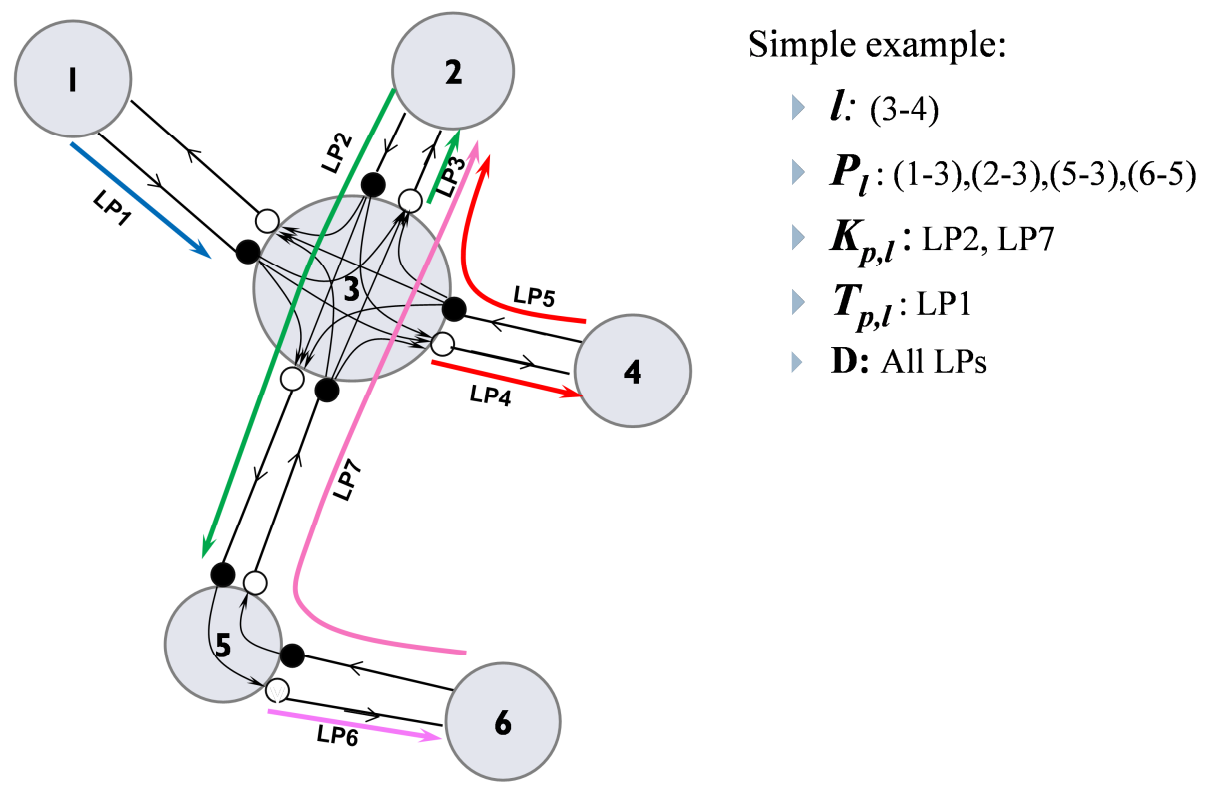

Figure 8. An illustrative example of the defined parameters

\subsubsection{Find}

$X_{n, l}^{\lambda}:$ is 1 if demand $n$ is routed through link $l$ on wavelength $\lambda$.

$C_{n}^{\lambda}$ : is 1 if $\lambda$ is used for demand $n$. 
$F_{n}^{\lambda}:$ is 1 if one filter is placed in destination node of demand $\mathrm{n}$ working on wavelength $\lambda$.

$W^{\lambda}:$ is 1 if wavelength $\lambda$ is in use.

$W L$ : total number of wavelengths used in the network.

\section{Minimize WL}

\subsection{Constraints}

While the objective of the problem is minimized, these constraints should be satisfied in order to get the optimal result. The proposed ILP model is subject to the following constraints for wavelength assignment and filter placement in passive optical backbone network design:

- Subject to:

$$
\begin{aligned}
& \sum_{n \in D} X_{n, l}^{\lambda}+\sum_{n \in K_{p, l}} X_{n, p}^{\lambda}+\left(\sum_{n \in T_{p, l}} X_{n, p}^{\lambda}-\sum_{n \in T_{p, l}} F_{n}^{\lambda}\right) \leq 1 \quad \forall l \in E, p \in P_{l}, \lambda \in w \\
& \sum_{n \in D} X_{n, l}^{\lambda}+X_{e, p}^{\lambda}-F_{e}^{\lambda} \leq 1 \quad \forall l \in E, e \in T_{p, l}, p \in P_{l}, \lambda \in w \\
& \sum_{n \in D} X_{n, l}^{\lambda}+\sum_{n \in K_{p, l}} X_{n, p}^{\lambda} \leq 1 \quad \forall l \in E, p \in P_{l}, \lambda \in w \\
& C_{n}^{\lambda} \geq F_{n}^{\lambda} \forall n \in T_{p, l}, \lambda \in w, p \in P_{l}, l \in E \\
& \sum_{\lambda \in w} F_{n}^{\lambda} \leq 1 \quad \forall n \in T_{p, l}, p \in P_{l}, l \in E \\
& \sum_{\lambda \in w} \sum_{n \in T} F_{n, l}^{\lambda}=N F \quad \forall p \in P_{l}, l \in E \\
& \sum_{\lambda \in w} X_{n, l}^{\lambda}=1 \quad \forall n \in D, l \in E \\
& C_{n}^{\lambda} \leq \sum_{l \in E} X_{n, l}^{\lambda} \forall n \in D, \lambda \in w \\
& C_{n}^{\lambda} \geq X_{n, l}^{\lambda} \forall n \in D, l \in E, \lambda \in w \\
& \sum_{\lambda \in w} C_{n}^{\lambda=1} \forall n \in D \\
& X_{n, l}^{\lambda}=X_{n, p}^{\lambda} \quad \forall n \in D, \lambda \in w, l \in E, p \in P_{l} \\
& \text { where destination node of } p \text { is source for } l \\
& W^{\lambda} \geq X_{n, l}^{\lambda} \forall n \in D, l \in E, \lambda \in w \\
& \sum_{\lambda \in w} W^{\lambda}=W L \\
& n
\end{aligned}
$$


Constraints (1-3) are considered in order to reuse wavelengths and avoid conflicts, where (1) and (2) are checking the possibility of having filters in the parent links. Constraint (3) ensures wavelength conflict avoidance, when there is no possibility to place filter in the parent links.

Constraints (4-6) guarantee that only one filter can be utilized for one demand and its filtered wavelength can be reused for the other demands.

Constraint (7) ensures that each demand can use only one wavelength.

Constraints (8-11) are employed for wavelength continuity.

Finally, constraints (12) and (13) are used to calculate wavelength usage. 


\section{Chapter4. Performance Evaluation}

To evaluate the proposed ILP formulation, a set of simulations were performed on 7-node subset of German network with a non-uniform traffic matrix and 10-node Italian network with a uniform traffic matrix [7]. The German topology has 3 fiber trees, 22 fiber links and 148 demands, while Italian network has 2 fiber trees, 30 fiber links and 90 demands. Figure 9 and Figure 10 show German and Italian networks. The model was evaluated in terms of wavelength usage, cost and energy consumption. In this study two passive OBN approaches are compared with the active OBN.

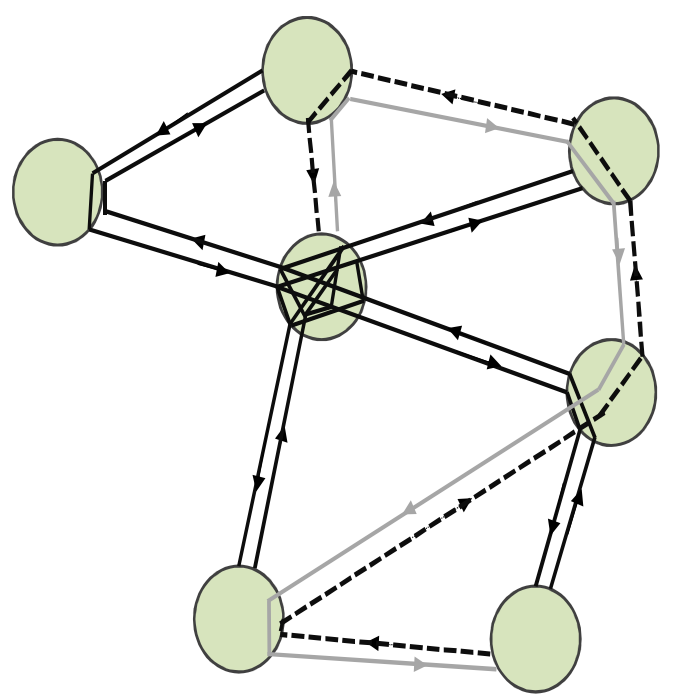

Figure 9. German network topology

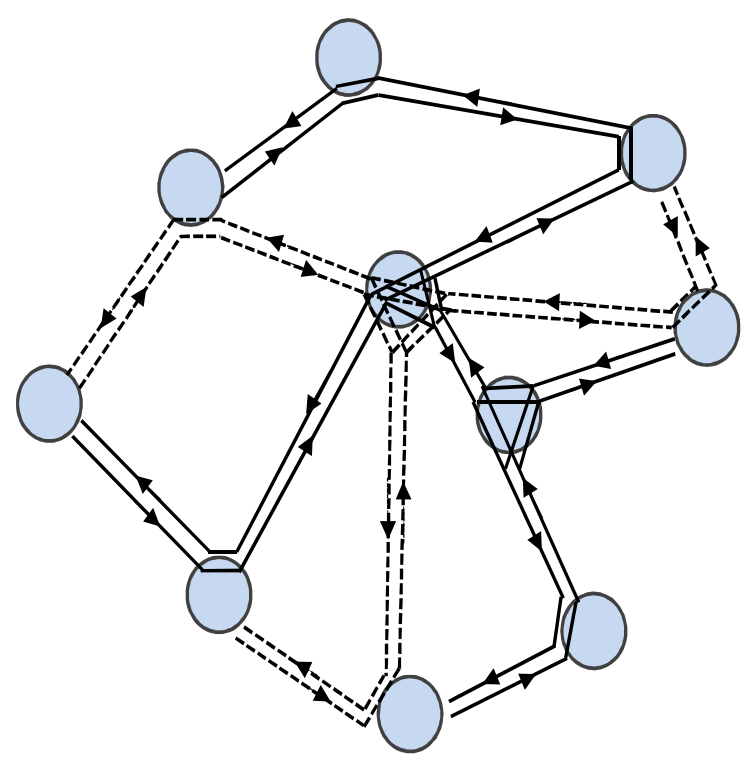

Figure 10. Italian network topology 


\subsection{Wavelength Usage}

Figure 11 shows the results of wavelength usage as a function of the number of optimally placed passive filters. As it is represented in the figure, a significant reduction in the number of wavelengths required to support the considered traffic demand, is obtained by applying passive filters. By increasing the number of filters in the network, the number of wavelengths is decreased to a certain point after which the curve is saturated. It means that placing more filters in the network will not further reduce the number of wavelengths.

This is an interesting observation which gives important information for the network provider regarding the number of filters that is beneficial to deploy. For the Italian network the maximum wavelength reuse can be attained with 12 filters and in German topology more than 26 filters are required. This difference arises due to different traffic matrices. As mentioned earlier, for the German network a non-uniform traffic matrix was considered while the Italian network used a uniform traffic matrix. It should be mentioned that the purpose of Figure 11 is not to compare the German and Italian topologies, but to show the trend in reaching the saturation point in different networks. It can be concluded that using uniform traffic, the saturation point can be reached utilizing smaller number of filters than the network with non-uniform traffic. The saturation point indicates the number of wavelengths is not further reduced by increasing the number of filters placed in the network.

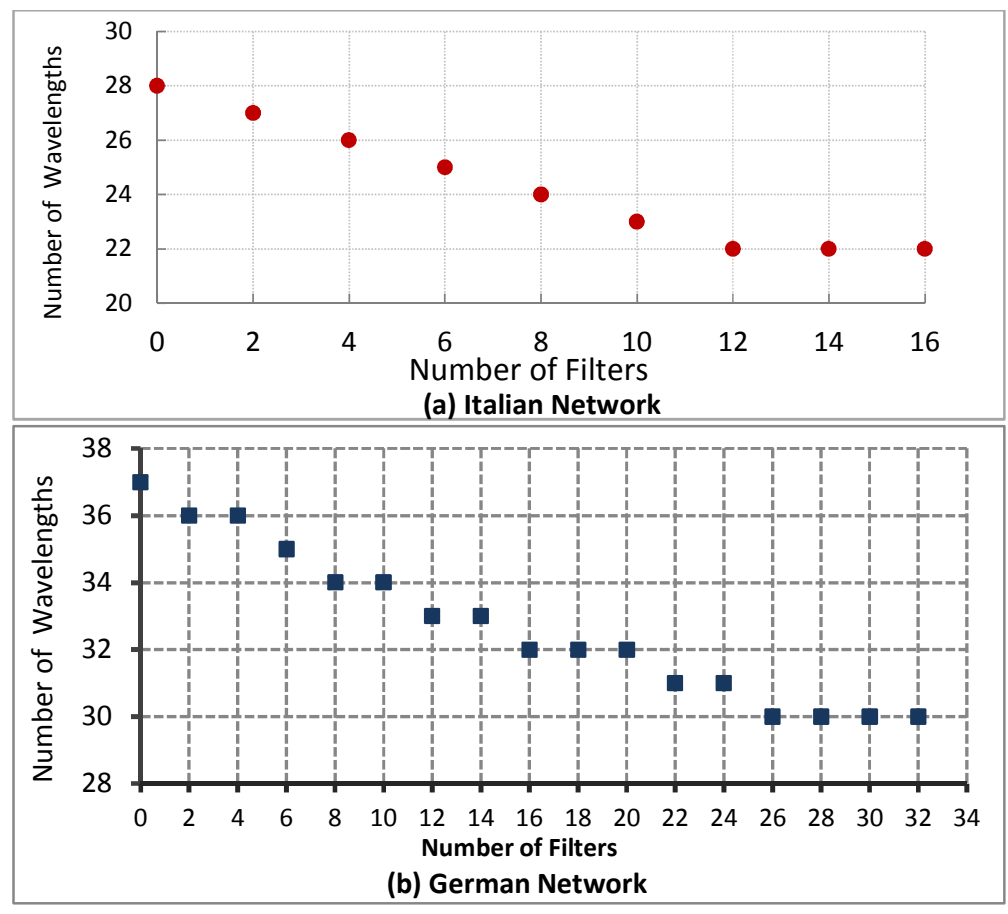

Figure 11. Wavelength usage vs. number of filters in 10-node Italian network (a) and 7-node German network (b) 
Figure 12 depicts the comparison between active photonic switch, filterless and semifilterless OBN architectures. Taking benefit of the proposed optimal model and placing some passive filters in the network, wavelength utilization has been improved in semifilterless approach, in comparison with the filterless network .

Furthermore, by solving the ILP model and finding the optimum filter placement, similar results are achieved for wavelength usage in SF-OBN with the active OBN. Results in Figure 12 shows that by using a set of passive filters in selected number of nodes, the disadvantage of filterless solution in terms of wavelength re-use can be overcomed.

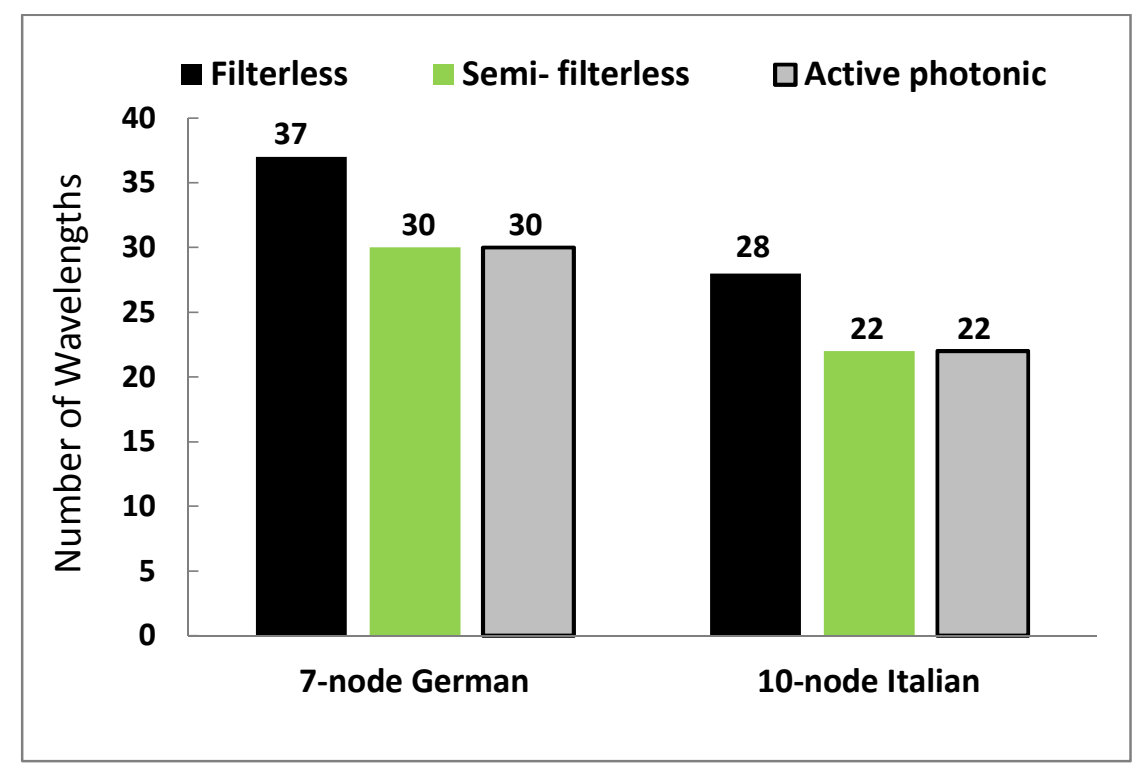

Figure 12. Wavelength utilization comparisons for active and passive architectures 


\subsection{Cost and Energy Comparisons}

The following models [10] were used for the cost and energy consumption by considering the normalized values for the components Error! Reference source not found.as shown in Table 2.

\section{- Active photonic:}

Total Cost $=N_{T-n o-e D C M} \cdot C_{T-n o-e D C M}+N_{W S S} \cdot C_{W S S}+N_{O L A} \cdot C_{D S}$

Total Power Consumption $=N_{T-n o-e D C M} \cdot P_{n o-e D C M}+N_{W S S} \cdot P_{W S S}+N_{O L A} \cdot P_{D S}$

\section{- Passive OBN (filterless and semi-filterless):}

Total Cost $=N_{T-e D C M} \cdot C_{e D C M}+N_{\text {Cou }} \cdot C_{\text {Cou }}+N_{O L A} \cdot C_{S S}+N_{F} \cdot C_{F}$

Total Power Consumption $=N_{T-e D C M} \cdot P_{n o-e D C M}+N_{O L A} \cdot P_{S S}$

Table 2. Normalized values for cost and energy consumption in arbitrary unit (a.u.)[11]

\begin{tabular}{|c|c|c|c|}
\hline \multicolumn{2}{|c|}{ Component } & Cost* & Power Consumption** \\
\hline \multicolumn{2}{|c|}{ Coupler(Cou) } & $\mathrm{C}_{\mathrm{cou}}=0.02$ & $\mathrm{P}_{\text {cou }}=0$ \\
\hline \multicolumn{2}{|c|}{ Filter(F) } & $\mathrm{C}_{\mathrm{F}}=0.035$ & $\mathrm{P}_{\mathrm{F}}=0$ \\
\hline \multirow[b]{2}{*}{$\begin{array}{c}\text { Optical Line } \\
\text { Ampilifier(OLA) }\end{array}$} & Single-Stage & $\mathrm{C}_{\mathrm{SS}}=1.3$ & $\mathrm{P}_{\mathrm{SS}}=1.3$ \\
\hline & Dual-Stage & $\mathrm{C}_{\mathrm{DS}}=2.6$ & $\mathrm{P}_{\mathrm{DS}}=1.8$ \\
\hline \multicolumn{2}{|c|}{ Wavelength Selective Switch (WSS) } & $\mathrm{C}_{\mathrm{WSS}}=2.5$ & $\mathrm{P}_{\mathrm{WSS}}=0.9$ \\
\hline \multicolumn{2}{|c|}{10 G Transponder with eDCM (T-eDCM) } & $\mathrm{C}_{\mathrm{T}-\mathrm{eDCM}}=1.2$ & $\mathrm{P}_{\mathrm{T}-\mathrm{eDCM}}=1$ \\
\hline \multicolumn{2}{|c|}{10 G Transponder without eDCM (T-no-eDCM) } & $\mathrm{C}_{\mathrm{T} \text {-no-eDCM}}=1$ & $\mathrm{P}_{\mathrm{T}-\mathrm{no}-\mathrm{eDCM}}=0.9$ \\
\hline
\end{tabular}

For the active photonic switching network, the following assumptions are taken:

- Two EDFAs per WSS are used

- Two EDFAs per OLA are required (DCF is neglected)

- WSS is required only in nodes with degree higher or equal to 3

The number of required WSS per node is given by:

$$
N_{W S S_{-} \text {Node }}=\left\{\begin{array}{c}
D \text { if } D>2 \\
0 \text { else }
\end{array}\right.
$$


Then, the number of required WSS per topology is (where $\mathrm{N}$ is the number of nodes):

$$
N_{W S S}=\sum_{i=0}^{i<N} N_{W S S_{-} N o d e \_} i
$$

$N_{W S S}$ : The number of WSS is degree dependent; therefore it is necessary to find how many nodes have a degree of a specific value. The number of WSS for German network is 4 and for the Italian network the value is equal to 6.

$N_{O L A}$ : Since the nodes and the links cost were included in the model the number of optical line amplifiers need to be considered. With a span length of $80 \mathrm{~km}$ in worst case the number of amplifiers required for the German and Italian networks are 26 and 36 respectively. In active photonic switching network dual stage OLA should be considered, which is equal to the number of WSS plus the number of OLAs.

Similar to the active network there are number of assumptions for passive network architecture which can be listed as follows:

- 1 EDFA per degree is needed

- 1 EDFA per OLA is required

- Number of passive couplers (e.g. passive optical splitter/combiner) is equal to the total number of degrees

Passive network uses single stage OLA and it is equal to the number of couplers plus the number of OLAs.

Moreover, it should be mentioned that active networks have transponders without dispersion compensation while passive architectures utilize transponder with dispersion compensation which consumes more energy.

Table 3 shows the number of elements which are used in the German and Italian networks.

Table 3. The number of components in the German and Italy networks

\begin{tabular}{|l|c|c|}
\cline { 2 - 3 } \multicolumn{1}{c|}{} & German 7 & Italy 10 \\
\hline $\mathbf{N}_{\text {WSS }}$ & 18 & 24 \\
$\boldsymbol{N}_{\boldsymbol{O L A}}$ & 26 & 36 \\
$\boldsymbol{N}_{\text {OLA }}(\boldsymbol{D S})$ & $18+26=44$ & $24+36=60$ \\
$\mathbf{N}_{\text {couplers }}$ & 22 & 30 \\
$\boldsymbol{N}_{\text {OLA }}(\boldsymbol{S S})$ & $22+26=48$ & $30+36=60$ \\
\hline
\end{tabular}

Figure 13 provides cost and energy consumption comparison for the three considered optical network approaches i.e. filterless, semi-filterless and active photonic networks. Since the ILP solution always finds the optimum value of number of wavelengths for the 
semi-filterless approach, it proves that it is possible to reach the same level of wavelength usage as in the active photonic case by placing a small number of filters. The results shown in Figure 13Error! Reference source not found. (a-b) demonstrate that the filterless and semi-filterless networks have significantly lower cost and power consumption as compared to the active photonic networks.
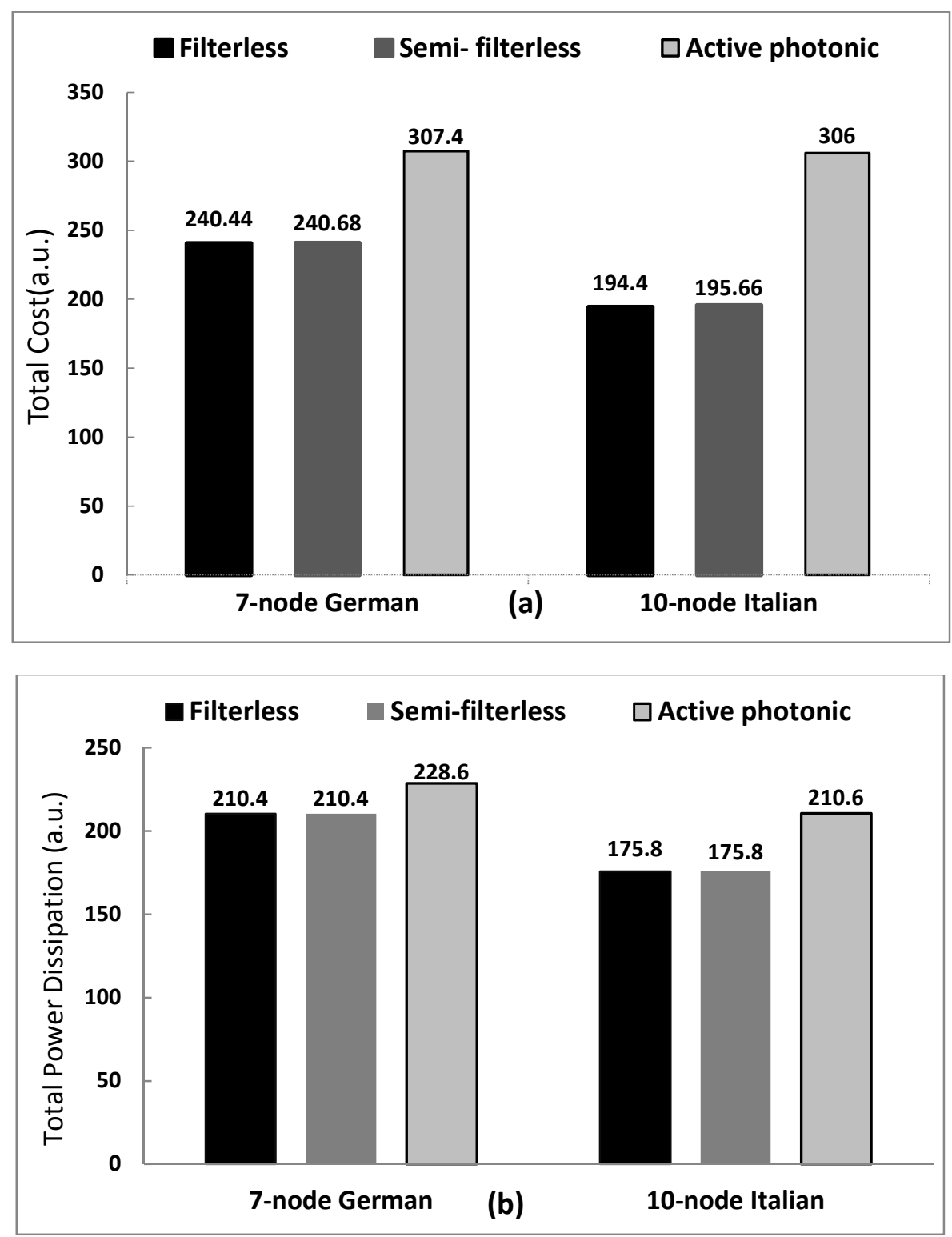

Figure 13 Cost (a) and energy consumption (b) comparisons for active and passive architectures 


\section{Chapter5. Conclusion and Future Work}

In this thesis project, an ILP model has been proposed along with optimal solutions for wavelength assignment and filter placement in passive optical backbone networks. The results reveal that the filterless OBN can be easily upgraded to the semi-filterless OBN, at the expense of a minor increase in investment costs, which can achieve the same level of efficiency in wavelength usage as the active switching networks. This in turn gives a potential for the higher scalability and better performance of semi-filterless networks as compared to the filterless approach. The results also confirm that passive OBN architectures achieve a significantly lower cost and power consumption as compared to their active counterparts.

For any further development and extension of the project, the ILP results can be used as a reference point for heuristic results comparison. It is recommended to extend the study and evaluate the performance of semi-filterless approach in terms of reliability and flexibility. 


\section{References:}

[1] B. Mukherjee, "Optical WDM Networks", New York: Springer, Verlag, 2006.

[2] J. Berthold, A.A.M. Saleh, L. Blair, and J.M. Simmons, IEEE/OSA Journal of Lightwave Technology, vol. 26, no. 9, pp. 1104-1118, 2008.

[3] C. Tremblay, et al, Technical Digest: 12th Opto-Electronics and Communications Conf., paper 12P-7, pp. 466-467, 2007.

[4] J. Chen, et al, in Proc. of IEEE ICTON 2011, Stockholm, Sweden, June 2011.

[5] S. Khanmohamadi, et al., in Proc. of ACP 2011, Shanghai, China, November 2011.

[6] É. Archambault, et al, Journal of Opt. Comm Netw., Vol. 2, No. 8, pp. 496 - 501, 2010.

[7] S. Gringeri, et al, IEEE Communications Magazine, July 2010.

[8] http://en.wikipedia.org/wiki/Linear_programming

[9] F. Abtahi, et al., in Proc. Of ACP 2012, Guangzhou, China, November 2012.

[10] Semi-filterless and filterless project input data, École de technologie supérieure (ÉTS), 2012.

[11] J. McNicol, et al., OFC'05, OThJ3 (2005). 


\section{Appendices}

Among our contributions to the overall project, the following articles have been published:

[1] Farzad Abtahi, Cicek Cavdar, Jiajia Chen, Sahar Khanmohamadi, Lena Wosinska, Guillaume Mantelet, Émile Archambault, Christine Tremblay and Michel P. Bélanger, "Optimal Design of Cost- and Energy-Efficient Scalable Passive Optical Backbone Networks", in Proc. of IEEE/OSA/SPIE Asia Communications and Photonics Conference and Exhibition (ACP), Nov. 2012.

[2]Sahar Khanmohamadi, Jiajia Chen, Farzad Abtahi, Lena Wosinska, Andrew Cassidy, émile Archambault, Christine Tremblay, Serge Asselin, Paul Littlewood, and Michel Bélanger, "Semifilterless optical network: a cost-efficient passive wide area network solution with effective resource utilization", in Proc. of IEEE/OSA/SPIE Asia Communications and Photonics Conference and Exhibition (ACP), Nov. 2011. (Furthermore, the article was nominated as the best student paper competition)

[3] Jiajia Chen, Sahar Khanmohamadi, Farzad Abtahi, Lena Wosinska, Zhenyu Xu, AndrewCassidy, Christine Tremblay, Paul Littlewood, Serge Asselin and Michel P. Bélanger, "PassiveWide AreaNetwork Solutions: Filterless and Semi-Filterless Optical Networks", in Proc. of IEEE International Conference on transparent Optical Networks ICTON2011, June 2011. 



\title{
Optimal Design of Cost- and Energy-Efficient Scalable Passive Optical Backbone Networks
}

\author{
Farzad Abtahi $^{1}$, Cicek Cavdar ${ }^{1}$, Jiajia Chen ${ }^{1}$, Sahar Khanmohamadi ${ }^{1,4}$, Lena Wosinska ${ }^{1}$, \\ Guillaume Mantelet $^{2}$, Émile Archambault ${ }^{2}$, Christine Tremblay ${ }^{2}$ and Michel P. Bélanger ${ }^{3}$ \\ (1) The Royal Institute of Technology KTH, Electrum 229, 16440 Kista, SWEDEN, abtahi@kth.se, \\ (2)École de technologie supérieure (ÉTS), 1100 Notre-Dame Ouest, Montréal, Québec H3C 1K3, \\ CANADA, (3)Ciena Corp., 3500 Carling Ave., Ottawa, Ontario, K2H 8E9, CANADA, (4) Ericsson \\ $A B$, Isafjordsgatan 10, 164 80, Kista, Sweden
}

\begin{abstract}
We propose an optimization model minimizing number of wavelengths in passive optical backbone networks and obtaining the same resource usage as in networks based on active switching while reducing both cost and power consumption.
\end{abstract}

OCIS codes: (060.0060) Fiber optics and optical communications; (060.4256)

Networks, network optimization; (060.4264) Networks, wavelength assignment

\section{Introduction}

Benefiting from advanced optical coherent transmission and electronic compensation technologies at the receiver, filterless $[1,2]$ optical backbone networks (FOBNs) can eliminate the need of active optical switching equipment in the network, using passive components, i.e., power splitters/combiners to interconnect fiber links. Consequently, FOBNs are more cost- and energyefficient than the networks based on active photonic switching devices. However, due to the broadcasting nature of FOBNs, resource reuse in the network can be very limited and hence the number of wavelengths required to support a certain traffic demand may be significantly higher than in the active networks. To alleviate this problem and keep the advantages of passiveness, the semi-filterless approach (S-FOBN), where passive filters are introduced in some selected nodes, has been introduced in [3, 4]. In this paper we present an optimum solution for both FOBN and SFOBN by developing an integer linear programming (ILP) model for the wavelength assignment and filter placement problems to minimize the cost. Our cost and power consumption models are more precise compared to $[3,4]$ where links and transponders were not taken into account.

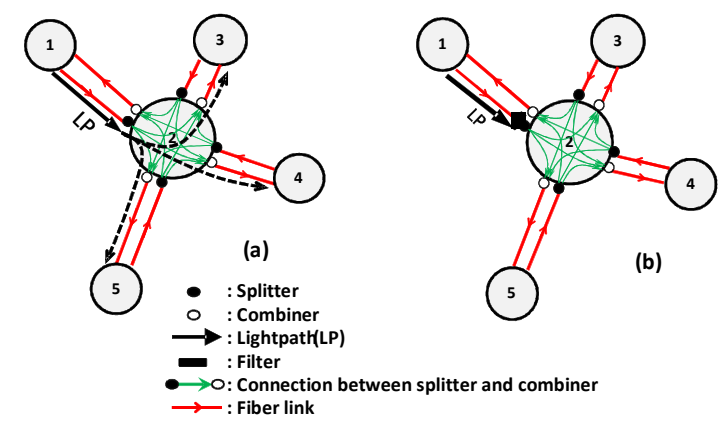

Fig. 1: Illustrative example: (a) FOBN (b) S-FOBN

One of the issues to be taken into account in passive OBNs is the laser effect caused by the amplifier gain in fiber loops, which need to be avoided at fiber interconnection design step [1]. Furthermore, in FOBN any wavelength assigned for a source destination pair can be used only once in a given branch of a fiber tree. Figure 1 illustrates an example of a simple fiber tree for filterless and semi-filterless networks. The directed fiber link from node 1 to node 2 is referred to as a parent of links between node 2-3, 2-4 and 2-5 (see Fig. 1(a)).The green lines inside node 2 represent the connections between splitters and combiners. As can be seen in Fig. 1 (a), without 
any filters all the lightpaths starting from node 1 and passing the 1-2 link will continue through all the child links, irrespectively if some of the lightpaths are addressed to node 2 and could be dropped there. However, in a semi-filterless network the wavelength of e.g. lightpath LP can be reused in any following child link because it is removed by the one colored passive filter placed in node 2 (see Fig. 1(b)).

The design of passive optical backbone network (OBN) can consist of three steps [1, 4]: (1) Fiber interconnection, (2) routing and (3) wavelength assignment and filter placement (for semifilterless network). Heuristic solutions are provided in [1,2] for filterless and in [4] for semifilterless approaches. In this paper we develop an integer linear programming (ILP) model minimizing number of wavelengths by filter placement in the network and give the optimum solution for S-FOBN. Moreover, in order to get the whole picture we quantified the benefits of the passive OBNs in terms of cost, wavelength usage and power consumption compare to the optical networks based on active photonic switching.

\section{Wavelength Assignment and Filter Placement Problem Formulation}

In this section, we present an ILP model for the design of S-FOBNs. The objective is to minimize the total number of wavelengths in the network by placing a given amount of passive filters in the selected nodes. Note that this model can also be used to optimize the number of wavelengths in FOBNs by setting the number of filters in the model equal to 0 . The problem formulation and ILP model is shown below.

- Given:

$D$ : the set of demands along with their routing information. $w$ : minimum number of wavelengths for each link.

$G(N, E)$ : the physical topology consisting the set of nodes $N$ and set of fiber links $E$ where $l \in E$ denotes one fiber link. $N F$ : the number of filters that need to be placed. $P_{l}$ : the set of parent links of $l$ where $P_{l} \subset E$ and $\mathrm{p}$ is used to index the parent links i.e., $p \in P_{l}$. $K_{p, l}$ : the set of demands which is routed through p but not $l$ where $K_{p, l} \subset D$. $T_{p, l}$ : the set of demands which is routed through $p$ and ended before $l$ where $T_{p, l} \subset D$.

- Find:

$X \underset{n, l}{\lambda}$ : is 1 if demand $n$ is routed through link $l$ on wavelength $\lambda$.

$C_{n}^{\lambda}:$ is 1 if $\lambda$ is used for demand $n$.

$F_{n}^{\lambda}:$ is 1 if one filter is placed in destination node of demand $\mathrm{n}$ working on wavelength $\lambda$.

$W^{\lambda}$ : is 1 if wavelength $\lambda$ is in use.

$W L$ : total number of wavelengths used in the network.

\section{Minimize $W L$}

- Subject to 


$$
\begin{array}{ll}
\sum_{n \in D} X_{n, l}^{\lambda}+\sum_{n \in K_{p, l}} X_{n, p}^{\lambda}+\left(\sum_{n \in T_{p, l}} X_{n, p}^{\lambda}-\sum_{n \in T_{p, l}} F_{n}^{\lambda}\right) \leq 1 \quad \forall l \in E, p \in P_{l}, \lambda \in w \\
\sum_{n \in D} X_{n, l}^{\lambda}+X_{e, p}^{\lambda}-F_{e}^{\lambda} \leq 1 \forall l \in E, e \in T_{p, l}, p \in P_{l}, \lambda \in w & \text { (2) } \sum_{n \in D} X_{n, l}^{\lambda}+\sum_{n \in K_{p, l}} X_{n, p}^{\lambda} \leq 1 \forall l \in E, p \in P_{l}, \lambda \in w \\
\mathrm{C}_{n}^{\lambda} \geq F_{n}^{\lambda} \forall n \in T_{p, l}, \lambda \in w, p \in P_{l}, l \in E & \text { (4) } \sum_{\lambda \in w} F_{n}^{\lambda} \leq 1 \forall n \in T_{p, l}, p \in P_{l}, l \in E \\
\sum_{\lambda \in w} \sum_{n \in T_{p, l}} F_{n}^{\lambda}=N F \forall p \in P_{l}, l \in E & \text { (6) } \sum_{\lambda \in w} X_{n, l}^{\lambda}=1 \forall n \in D, l \in E \\
C_{n}^{\lambda} \leq \sum_{l \in E} X_{n, l}^{\lambda} \forall n \in D, \lambda \in w & \text { (8) } C_{n}^{\lambda} \geq X_{n, l}^{\lambda} \forall n \in D, l \in E, \lambda \in w \\
X_{n, l}^{\lambda}=X_{n, p}^{\lambda} \forall n \in D, \lambda \in w, l \in E, p \in P_{l} & \text { where destination node of } p \text { is source for } l \\
\sum_{\lambda \in w} C_{n}^{\lambda}=1 \forall n \in D & \text { (11) } W^{\lambda} \geq X_{n, l}^{\lambda} \forall n \in D, l \in E, \lambda \in w \\
\sum_{\lambda \in w} W^{\lambda}=W L & \text { (13) }
\end{array}
$$

In order to reuse wavelengths and avoid conflicts, constraints (1-3) are considered, where (1) and (2) are checking the possibility of having filters in the parent links and constraint (3) ensures wavelength conflict avoidance, when there is no possibility to place filter in the parent links. Constraints (4-6) guarantee that only one filter can be utilized for one demand and its filtered wavelength can be reused for the other demands. Constraint (7) ensures that each demand can use only one wavelength. Constraints (8-11) are employed for wavelength continuity. Finally, constraints (12) and (13) are used to calculate wavelength usage.

\section{Results}

To evaluate the proposed ILP formulation we performed a set of simulations on different networks. As an example, we present the results for 10-node Italian network with a uniform traffic matrix1 with a set of 90 demands each of which has granularity of $10 \mathrm{Gbps}$. The results obtained for other networks show similar benefit achieved by our optimization as the ones presented here for the Italian network. Figure 2(a) shows the results of wavelength usage as a function of the number of optimally placed passive filters. It can be seen that a significant reduction of number of wavelengths required to support the considered traffic demand is obtained by applying passive filters. The number of wavelengths needed is decreasing with increasing number of passive filters up to the certain point. Then, the curve is saturated, which means that placing more filters in the network will not further reduce the number of wavelengths. This is giving important information for the network provider regarding the number of filters that is beneficial to deploy. For the Italian network the maximum wavelength reuse can be attained with 12 filters. Figures 2 (b-d) provide a performance comparison in terms of wavelength utilization, cost and energy consumption for the three considered network approaches, i.e. filterless, semi-filterless and active photonic networks. Since ILP solution always finds the optimum value of number of wavelengths for semi-filterless approach, it proves that it is possible to reach the same level as in the active photonic case by placing a small number of filters. The results shown in Fig. 2(c-d) demonstrate that the filterless and semi-filterless networks have significantly lower cost and power consumption compared to the active photonic networks. 


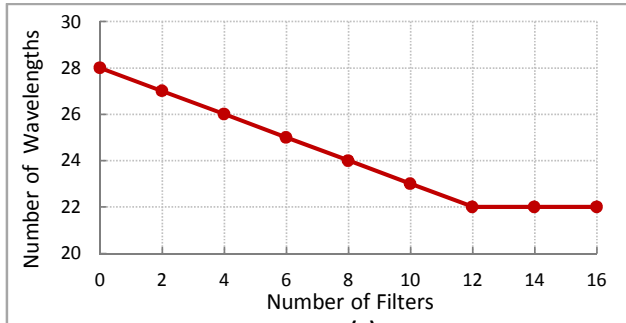

(a)

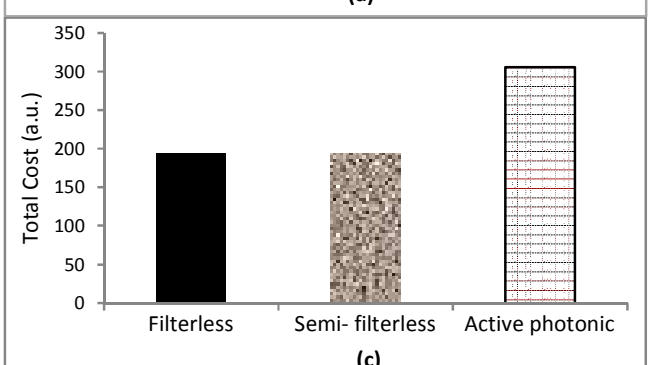

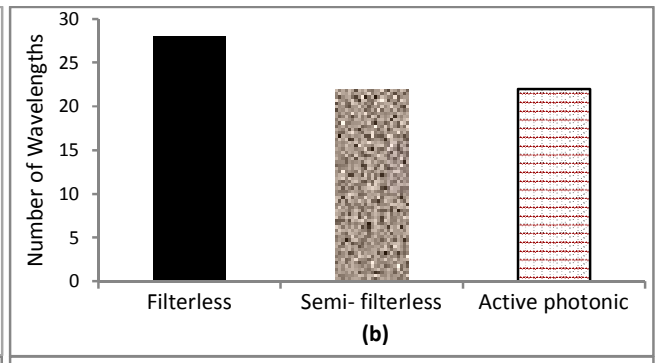

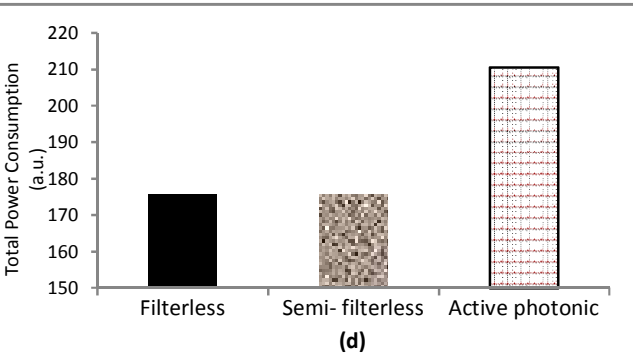

Fig. 2: (a) Number of wavelengths vs. number of filters in passive OBN and performance comparison for filterless, semi-filterless and active photonic solutions in terms of (b) number of wavelengths, (c) cost and (d) power consumption

We used the following models for the cost and energy consumption by considering the normalized values for the components which are shown in Tab. 1. In the formulations (14-17) $\mathrm{N}$ indicates number of specific devices while $\mathrm{C}$ and $\mathrm{P}$ represent cost and power consumption of corresponding devices, respectively. In passive OBNs we considered transponders with electronic dispersion compensation module (T-eDCM) and single stage (SS) optical line amplifiers (OLA) while in active architecture we used transponders without eDCM (T-no-eDCM) together with dual stage (DS) OLAs, due to longer transmission distance and higher impairment in passive OBNs.

- Active photonic:

Total Cost $=N_{T-n o-e D C M} \cdot C_{T-n o-e D C M}+N_{W S S} \cdot C_{W S S}+N_{O L A} \cdot C_{D S}$

Total Power Consumption $=N_{T-n o-e D C M} \cdot P_{n o-e D C M}+N_{W S S} \cdot P_{W S S}+N_{O L A} \cdot P_{D S}$

- Passive (filterless and semi-filterless):

Total Cost $=N_{T-e D C M} \cdot C_{e D C M}+N_{\text {Cou }} \cdot C_{C o u}+N_{O L A} \cdot C_{S S}+N_{F} \cdot C_{F}$

Total Power Consumption $=N_{T-e D C M} \cdot P_{n o-e D C M}+N_{O L A} \cdot P_{S S}$

Tab. 1: Normalized values for cost and energy consumption in arbitrary unit (a.u.) [5]

\begin{tabular}{|c|c|c|c|}
\hline \multicolumn{2}{|l|}{ Component } & Cost* $^{*}$ & Power Consumption** \\
\hline \multicolumn{2}{|l|}{ Coupler(Cou) } & $\mathrm{C}_{\text {cou }}=0.02$ & $\mathrm{P}_{\mathrm{cou}}=0$ \\
\hline \multicolumn{2}{|l|}{ Filter(F) } & $\mathrm{C}_{\mathrm{F}}=0.035$ & $\mathrm{P}_{\mathrm{F}}=0$ \\
\hline \multirow{2}{*}{ Optical Line Ampilifier(OLA) } & Single-Stage & $\mathrm{C}_{\mathrm{SS}}=1.3$ & $\mathrm{P}_{\mathrm{SS}}=1.3$ \\
\hline & Dual-Stage & $\mathrm{C}_{\mathrm{DS}}=2.6$ & $\mathrm{P}_{\mathrm{DS}}=1.8$ \\
\hline \multicolumn{2}{|c|}{ Wavelength Selective Switch (WSS) } & $\mathrm{C}_{\mathrm{wsS}}=2.5$ & $\mathrm{P}_{\mathrm{WSS}}=0.9$ \\
\hline \multicolumn{2}{|c|}{10 G Transponder with eDCM (T-eDCM) } & $\mathrm{C}_{\mathrm{T}-\mathrm{eDCM}}=1.2$ & $\mathrm{P}_{\mathrm{T}-\mathrm{eDCM}}=1$ \\
\hline \multicolumn{2}{|c|}{10 G Transponder without eDCM (T-no-eDCM) } & $\mathrm{C}_{\mathrm{T} \text {-no-eDCM}}=1$ & $\mathrm{P}_{\mathrm{T}-\mathrm{no}-\mathrm{eDCM}}=0.9$ \\
\hline
\end{tabular}

* Normalized to the cost of T-no-eDCM. **Normalized to the power consumption of T-eDCM. 
Filterless and semi-filterless line systems also exhibit lower power dissipation, thanks to the electronic dispersion compensation capability of the tunable receivers, which allows using singlestage optical amplifiers except at equalization sites. Calculations made for Italian network topology (80-km spans and two equalization sites) indicate savings of about $22 \%$ for optical links equal to the network diameter.

\section{Conclusion}

In this paper, an ILP model and optimal solutions have been proposed for wavelength assignment and filter placement in passive core networks. The results reveal that at the expense of the minor increase in investment cost the filterless approach can be easily upgraded to the semi-filterless solution which can achieve the same level of efficiency in wavelength usage as the active switching network. This in turn gives a potential for the higher scalability performance of S-FOBN compared to FOBN. Our results also confirmed that passive OBN architecture achieves lower cost and power consumption compared with their active counterpart.

\section{References}

[1] É. Archambault, et al, Journal of Opt. Comm. Netw., Vol. 2, No. 8, 496 - 501 (2010).

[2] C. Tremblay, et al, Technical Digest: 12th Opto-Electronics and Communications Conf., paper 12P-7, pp. 466-467 (2007).

[3] J. Chen, et al, in Proc. of IEEE ICTON 2011, Stockholm, Sweden, June 2011.

[4] S. Khanmohamadi, et al., in Proc. of ACP 2011, Shanghai, China, November 2011.

[5] J. McNicol et al., OFC'05, OThJ3 (2005). 


\title{
Semi-filterless optical network: a cost-efficient passive wide area network solution with effective resource utilization
}

\author{
Sahar Khanmohamadi ${ }^{1}$, Jiajia Chen ${ }^{1}$, Farzad Abtahi ${ }^{1}$, Lena Wosinska ${ }^{1}$, Andrew Cassidy ${ }^{2}$, \\ Émile Archambault ${ }^{2}$, Christine Tremblay ${ }^{2}$, Serge Asselin ${ }^{3}$, Paul Littlewood ${ }^{3}$, and Michel \\ Bélanger $^{3}$ \\ ${ }^{1}$ The Royal Institute of Technology KTH, Electrum 229, 16440 Kista, SWEDEN \\ ${ }^{2}$ École de technologie supérieure (ÉTS), 1100 Notre-Dame Ouest, Montréal, Québec H3C 1K3, \\ CANADA \\ ${ }^{3}$ Ciena Corp., 3500 Carling Ave., Ottawa, Ontario, K2H 8E9, CANADA \\ $\mathbf{1}^{\text {st }}$ author is a student
}

\begin{abstract}
By utilizing advanced optical coherent transmission and electrical compensation technologies, two novel passive wide area network (WAN) solutions, filterless and semi-filterless optical networks, are able to eliminate the usage of the expensive active photonic reconfigurable components by interconnecting the nodes with passive optical power splitters/combiners and using tunable transceivers. Therefore, they have a potential to be more cost-effective and energy-efficient as well as more reliable than the networks based on active optical switching. In comparison with the filterless approach, the semi-filterless optical network can improve resource utilization by introducing passive wavelength filters at some selected nodes. This paper proposes a semi-filterless optical network design tool for filter placement and wavelength assignment and validates it on a number of network topologies. Performance evaluation confirm that the proper design of semifilterless optical networks can offer significant reduction of the number of wavelengths needed to support a certain traffic demand compared to filterless networks while keeping all the advantages of the passive WAN solution.
\end{abstract}

Keywords: Passive wide area network, semi-filterless optical network,filter placement, wavelength assignment

\section{Introduction}

The exploration of two novel passive wide area network (WAN) solutions, namely, filterless $[1,2]$ and semi-filterless [3] optical networks has been stimulated by advances in optical coherent transmission as well as electrical compensation technologies. These two novel WAN approaches eliminate the usage of the active photonic reconfigurable component by utilizing passive optical power splitters/combiners, which makes this network architecture more cost-effective and energyefficient as well as more reliable compared with the networks based on active optical switching. 
On the other hand, the filterless optical network $[1,2]$ suffers from a constraint of wavelength reuse due to its broadcast characteristic resulting in wavelength continuity going further than the intended destination node and consequently any assigned wavelength can only be used once in a given fiber tree. As a result of this nature, the filterless solution always requires more wavelengths in order to satisfy the same traffic demands than the approach based on active optical switching. To address this issue, the concept of a semi-filterless optical network was proposed in [3] as an improvement and extension of the filterless solution. By introducing passive colored components - e.g., fiber Bragg gratings (FBG), red/blue filters, etc.- at some selected nodes in the fiber tree and utilizing its non-broadcast property to relax the wavelength reuse constraint inherent in the filterless approach, the semi-filterless network, therefore has an advantage to improve the wavelength utilization at a relatively low deployment cost. In this paper, a semi-filterless optical network design tool is proposed and validated on a number of network topologies. A comparison of cost and wavelength utilization in optical networks based on active switching, filterless and semi-filterless approach is provided.

\section{Proposed design tool for semi-filterless optical network}

The semi-filterless design problem can be partitioned into three main parts (see Figure 1): (1) Fiber interconnection, (2) routing and (3) filter placement and wavelength assignment (WA). As an extension of the filterless solution, semi-filterless optical networks can also take advantage of advanced modulation formats, electronic dispersion compensation and tunable transceivers to provide agility at network nodes and interconnect the nodes with passive splitters/combiners. Therefore, the schemes of physical link interconnection and lightpath routing used in the filterless optical network can be applied to the semi-filterless approach as well. Our semi-filterless design tool uses the efficient fiber connection and routing algorithms as in [1] for the first two steps considering similar input parameters and constraints for network design and planning. In contrast to the filterless solution, in a semi-filterless optical network, passive colored components (e.g., FBG, red/blue filters, etc.) are allowed to be placed at some selected nodes to drop the signal. In this way, the dropped wavelength can be reused for the lightpaths in the same fiber tree starting at or after the nodes equipped with the filter.

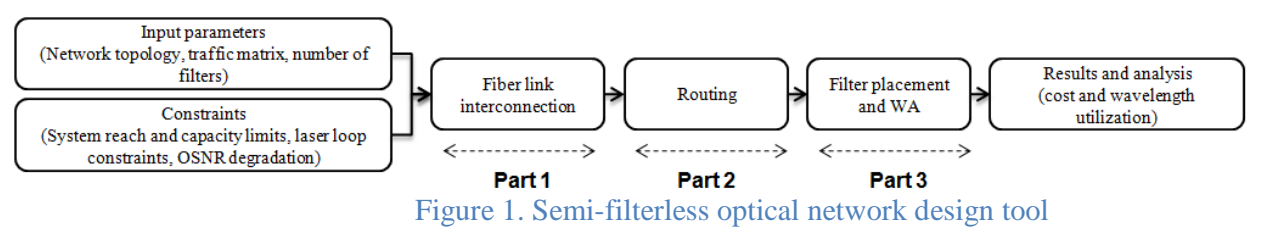

Figure 2 illustrates an example for the filterless and semi-filterless networks and the corresponding conflict graph for the wavelength assignment. The correlation between lightpaths [4] is shown in a 
conflict graph, where an edge represents wavelength clash constraint, i.e. the corresponding two lightpaths are not able to be assigned a common wavelength. Furthermore, we define the contact as the incoming or outgoing port for the nodes. In Fig.2, Node 2 has two incoming contacts, i.e. from Node 1 and 5, and two outgoing contacts to Node 3 and 4. Obviously, it is more efficient to place the filters to drop the wavelength at the incoming contact than at the outgoing contact. The filterless solution showed in Fig. 2 (a) needs at least three wavelengths for all four of the considered lightpaths. However, by introducing a filter at the incoming contact of Node 2 connecting to Node 1 to drop the wavelength signal of LP1 (see Fig.2 (b)), the number of the required wavelengths is reduced to 2 .

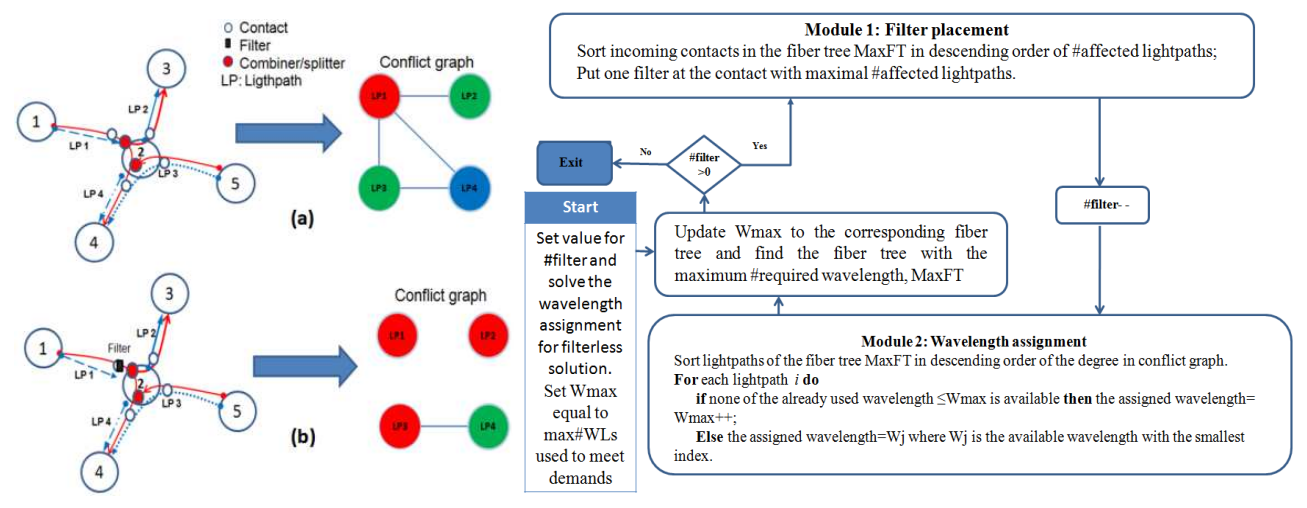

Figure 2. Illustrative example: filterless (a) vs. semi-filterless (b)
Figure 3. Flow chart of the proposed heuristic for filter placement and wavelength assignment.

The third step of our design tool called filter placement and wavelength assignment (WA) is an important part improving the wavelength utilization. We propose a heuristic algorithm with the objective to minimize the number of required wavelengths by placing a given number of filters. Figure 3 shows the flow chart of our algorithm. The whole process consists of several iterations (whose number is equal to the number of filters). In each round, two modules, called filter placement and wavelength assignment, are preceded. Typically, there are several fiber trees in the network. In the filter placement module, a fiber tree with the maximum number of required wavelengths is selected. Then, one filter is placed at the incoming contact with the maximal number of affected lightpaths in that fiber tree. In this way, a large number of edges in the related conflict graph can be removed which implies the potential of decreasing the number of assigned wavelengths. Furthermore, the greedy algorithm proposed in [4] is applied to the wavelength assignment module. In each iteration, only the fiber tree with the newly placed filter is considered. A conflict graph is generated accordingly and the wavelengths are assigned to the lightpaths according to the descending order of their degree. 


\section{Performance evaluation}

We implemented our heuristic scheme in $\mathrm{C}++$ and tested it on three different network topologies: 7-node subset of the German network, 10-node Italian network, and 17-node German network [1]. Furthermore, for simulation we considered a non-uniform traffic for 7-node German network and a uniform traffic for 10-node Italian network and 17-node German network as presented in [1]. Figure 4 (a-c) shows the number of required wavelengths as a function of the number of filters placed in the network for the considered network topologies. Obviously, the more filters the semifilterless solution has, the less number of wavelengths is required. However, after a certain point the curve becomes saturated. It means the number of wavelengths is not further reduced by increasing the number of filters placed in the network. The congestion represents the lower bound for the number of required wavelengths. For example, adding 40 filters in the 17-node German network can cause 20 percent reduction of the required number of wavelengths (see Fig. 4 (c)), whereas increasing the number of filters to more than 40 does not improve the wavelength utilization. Besides, it should be noted that the gain on wavelength reduction by introducing the filters is also dependent on the network topologies and traffic matrix. 10-node Italian and 17-node German network, which have uniform traffic, obtain better improvement of wavelength utilization than 7-node German network.

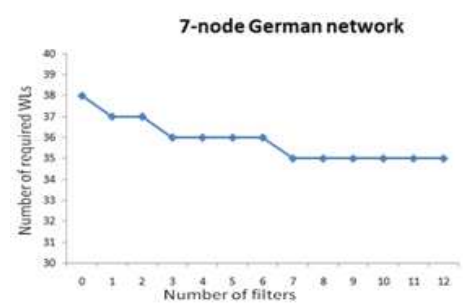

(a)

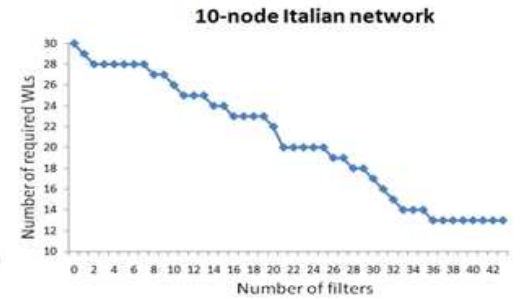

(b)

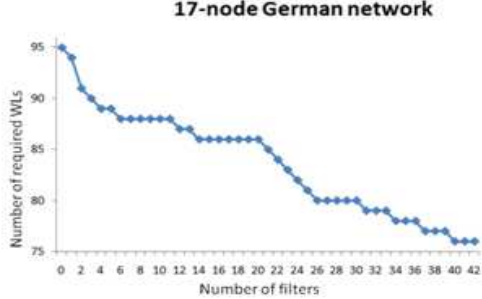

(c)

Figure 4. Number of wavelengths (WLs) vs. number of filters

Figure 5 shows the cost comparison of the three considered network scenarios where we assumed relative cost with arbitrary units, (a.u.). Moreover, we focus on the cost of nodes, since the cost of link is the same for different types of optical networks. The input data for each relative cost is obtained from [1] and [5]. In comparison with optical network based on active switching, both filterless and semi-filterless solutions have significantly lower cost for nodes since only passive components are required. Table 1 summarizes performance comparison in terms of cost and wavelength usage for all the considered scenarios. It is shown that the semi-filterless network can significantly improve the wavelength utilization at the expense of a minor increase of investment cost. 


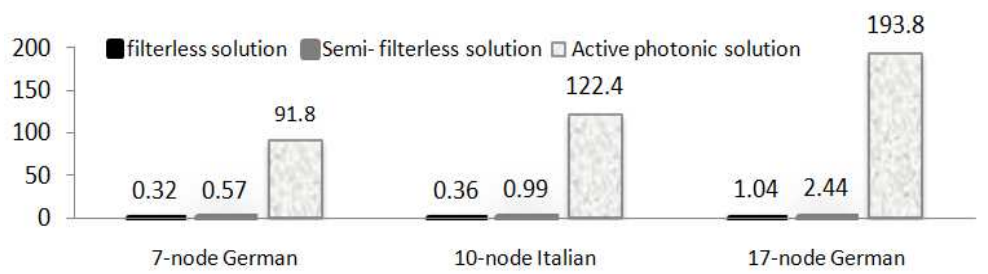

Figure 5. Cost comparison of filterless, semi-filterless and active photonic switching solution

Table 1: Performance comparison for filterless, semi-filterless and active photonic solutions

\begin{tabular}{|c|c|c|c|c|c|c|}
\hline \multirow{2}{*}{ Solutions } & \multicolumn{2}{|c|}{7 -node German } & \multicolumn{2}{l|}{ 10-node Italian } & \multicolumn{2}{l|}{ 17-node German } \\
\cline { 2 - 7 } & \#WLs & Cost & \#WLs & Cost & \#WLs & Cost \\
\hline Filterless & 37 & 0.32 & 28 & 0.36 & 88 & 1.04 \\
\hline Semi-filterless & 34 & 0.57 & 22 & 0.99 & 76 & 2.44 \\
\hline Active photonic & 30 & 91.8 & 22 & 122.4 & 56 & 193.8 \\
\hline
\end{tabular}

\section{Conclusion}

In this paper, an efficient semi-filterless network design tool has been proposed and validated on different network topologies. In comparison with active switching and filterless optical networks, it is shown that semi-filterless network is a cost efficient alternative with effective resource utilization. In order to obtain a comprehensive analysis for both passive and active optical WANs we plan to extend our study and evaluate other performance parameters, such as resilience, flexibility, power efficiency, etc.

\section{References}

[1] É. Archambault, et al, Journal of Opt. Comm Netw., Vol. 2, No. 8, pp. 496 - 501, 2010.

[2] C. Tremblay, et al, Technical Digest: 12th Opto-Electronics and Communications Conf., paper 12P-7, pp. 466-467, 2007.

[3] J. Chen, et al, in Proc. of IEEE ICTON (13th International Conference on Transparent Optical Networks), 2011.

[4] D. J. A. Welsh, et al, The Computer Journal , Vol. 10, No. 1, pp. 85-86, 1967.

[5] OASE ("OPTICAL ACCESS SEAMLESS EVOLUTION", a large scale Integrated Project funded by the European Commission through the 7th ICT-Framework Programme), private communication, 2011. 


\section{Passive Wide Area Network Solutions: Filterless and Semi-Filterless Optical Networks \\ Jiajia Chen ${ }^{1}$, Sahar Khanmohamadi ${ }^{1}$, Farzad Abtahi ${ }^{1}$, Lena Wosinska ${ }^{1}$, Zhenyu $\mathrm{Xu}^{2}$,Andrew Cassidy $^{2}$, Christine Tremblay ${ }^{2}$, Paul Littlewood ${ }^{3}$, Serge Asselin $^{3}$ and Michel P. Bélanger ${ }^{3}$ \\ 1 The Royal Institute of Technology KTH, Electrum 229, 16440 Kista, Sweden \\ 2 École de Technologie Supérieure, 1100 Notre-Dame Ouest, Montréal, Québec H3C 1K3, Canada \\ 3 Ciena, Canada, 3500 Carling Ave, Ontario, K2H 8E9, Canada \\ E-mail: jiajiac@kth.se}

\section{EXTENDED ABSTRACT}

Advances in optical coherent transmission and electrical compensation technologies (such as coherent receiver and forward error correction FEC) have stimulated ideas for novel optical network architectures. Recently proposed passive wide area network solution, referred to as filterless optical network [1-2] eliminates or minimizes the usage of active photonic reconfigurable network elements. In this approach, only the passive splitters and combiners for interconnecting the fiber links are utilized, which makes this network architecture more cost- and energy-effective as well as more reliable compared with networks based on active optical switching.

However, the filterless optical network architecture implies some constraints on fiber interconnection design, maximum fiber-tree length and wavelength reuse due to its broadcast nature. Consequently, filterless solution always requires more resources (i.e. number of wavelengths) compared with the active switched optical networks which are allowed to utilize reconfigurable and coloured components. In order to improve the wavelength utilization while maintaining flexibility of resource allocation, this work extends the idea of filterless optical network by introducing some passive coloured components (e.g., fiber Bragg grating FBG, red/blue filters, etc) to drop local signals at some determined nodes. This approach is referred to as semi-filterless optical network. Furthermore, the semi-filterless solution maintains the passive feature, enabling high reliability and efficiency of cost and energy. Meanwhile, its non-broadcast property at some determined nodes has potential to decrease the transmission impairments and hence relax the constraints on fiber interconnection design and the maximal transparent length, which are strict in the filterless optical network. Our preliminary results confirm the advantages of semifilterless solution.

Keywords: passive wide area network, filterless optical network, semi-filterless optical network.

\section{ACKNOWLEDGEMENTS}


This work was supported by project "Performance study of filterless and active switched optical networks",funded by Ciena, Canada.

\section{REFERENCES}

[1] C. Tremblay, F. Gagnon, B. Châtelain, É. Bernier, M. P. Bélanger, "Filterless optical networks: A unique

and novel passive WAN network solution", in Technical Digest: 12th Opto-Electronics and Communications Conf., 16th Int. Conf. on Integrated Optics and Optical Fiber Communication, OECC/IOOC, Kanagawa, Japan, 2007.

[2] É. Archambault, D. O'Brien, C. Tremblay, F. Gagnon, M. P. Bélanger, É. Bernier, "Design and simulation of filterless optical networks: Problem definition and performance evaluation”, Journal of Opt. Comm. Netw., vol. 2, no. 8, pp. 496-501, August 2010. 